\title{
Expression of a modified astrocytic glutamate transporter alleviates H untington's hypokinesia, promotes synaptic glutamate clearance and counteracts potentially adverse EAAT 2 interactions
}

Stefan Hirschberg*1, A nton D vorzhak*¹, Seyed M. A . Rasooli-N ejad ${ }^{1}$, Svilen A ngelov ${ }^{1}$, M arieluise Kirchner ${ }^{2,3}$, Philip Mertins ${ }^{2,3}$, Gilla Lättig-Tünnemann ${ }^{4,5}$, Christoph Harms ${ }^{4,5}$, Dietmar Schmitz ${ }^{6,7,8}$ and Rosemarie Grantyn $n^{1,4,7,8}$

*These authors equally contributed to the present work

1 Synaptic Dysfunction Lab, Neuroscience Research Center, Charité-Universitätsmedizin B erlin, Corporate member of Freie Universität B erlin, Humboldt-Universität zu Berlin, and B erlin Institute of Health, 10115, B erlin, Germany, stefan.hirschberg@ charite.de, anton.dvorzhak@charite.de

2 Proteomics Platform, Max Delbrück Center for Molecular Medicine in the Helmholtz Association, 13125 B erlin, Germany

3 B erlin Institute of Health (BIH), 13125 B erlin, Germany

4 Department of Experimental N eurology, Charité-Universitätsmedizin B erlin, 10117 B erlin, Germany

5 Center for Stroke Research B erlin, Charité-U niversitätsmedizin B erlin, 10117 B erlin, Germany

6 German Center for N eurodegenerative Diseases (DZNE), 10117 B erlin, Germany

7 Cluster of Excellence N euroCure, 10117, Berlin, Germany

8 Einstein Center for N eurosciences B erlin, 10117, B erlin, Germany, rosemarie.grantyn@ charite.de

\section{ACK NOWLEDGEMENTS}

Thanks are due to Profs. A rnd B aumann and Christoph Fahlke, ForschungszentrumJ ülich for the EY FP EAAT2 S506X plasmid, Prof. Francisco Zafra, Universidad Autónoma de Madrid, for important information on the 4K R-edited EAAT2 isoform, Dr. K. Török, St. George's, University of London, and Dr. N. Helassa, University of Liverpool, for the iGlu $u_{u}$ plasmid. Prof. Gudrun A hnert-Hilger, Charité Universitätsmedizin B erlin, and Dr. Hannes Schmidt, Eberhard Karls U niversität Tübingen, contributed numerous helpful suggestions. D. B etances and A. Schönherr provided excellent technical assistance. This work was supported by CHDI (A-12467), the German Research Foundation, under Germany's Excellence Strategy (Exc 2049 - 390688087) and intramural Charité Research Funds to R.G.

\section{SUM M ARY}

Rapid removal of glutamate from the sites of glutamate release is an essential step in excitatory synaptic transmission. Despite many years of research, the molecular mechanisms underlying the intracellular regulation of glutamate transport at tripartite synapses have remained unclear. This limits the options for pharmacological treatment of motor disorders associated with glutamate excitotoxicity. Therefore, using the Q175 mouse model of Huntington's disease (HD), we explored the effects of structural changes in the astrocytic excitatory amino acid transporter type 2 (EAAT 2). We report that expression of a C-terminalmodified variant of EAAT 2 can alleviate the symptoms of hypokinesia in mice with already advanced HD. At a cellular level, this beneficial outcome correlated with faster synaptic glutamate clearance, higher astrocytic glutamate uptake and larger amounts of native EAAT 2 protein. Proteomics data indicate a partial reversal of HD-induced changes in the EAAT2 interactor spectrum. Thus, astrocytic glutamate transport remains a target for therapeutic intervention.

\section{KEYWORDS}

Huntington's disease; EAAT2 interaction proteomics; Hypokinesia; Corticostriatal synaptic transmission; Glutamate clearance; Synaptic targeting of EAAT2 


\section{INTRODUCTION}

$H D$ is an autosomal dominant neurodegenerative disease. A trinucleotide (CAG) expansion of exon 1 in the mutant huntingtin gene (mHTT) causes abnormally long polyglutamine stretches in the already large huntingtin protein. $\mathrm{mHTT}$ is expressed not only in neurons but also in astrocytes (Faideau et al., 2010) where the loss of normal huntingtin function and additional effects of misfolded polyQ mHTT fragments can produce a large variety of alterations (M rzljak and M unoz-Sanjuan, 2015;Zeitler et al., 2019;Tabrizi et al., 2019). HD patients suffer from progressive motor, cognitive and emotional disturbances (Tabrizi et al., 2013). A prominent motor symptom of advanced HD in humans and most rodent models of the disease is hypo-/bradykinesia (B erardelli et al., 1999; Hart et al., 2013; Horton et al., 2019), i.e. a reduced incidence and speed of self-induced movements. The appearance of jerky uncontrolled spontaneous movements (chorea) is the predominant feature at earlier stages of HD (Rosenblatt et al., 2003;Tabrizi et al., 2013). Thorough quantification of the hypovs. hyperkinetic aspects of motor performance is important for the pharmacological management of the disease (Hart et al., 2013). In general, phenotype progression is less faithfully predicted by the scores of chorea as compared to those of hypo-/bradykinesia (Rosenblatt et al., 2003;T abrizi et al., 2013).

According to the information available from extracellular recordings or imaging of neuronal activity in intact HD mice, motor symptoms may evolve as a consequence of disinhibition and abnormal synchronicity in the corticostriatal pathway (Miller et al., 2012;Burgold et al., 2019). However, most studies point to functional uncoupling rather than enhanced glutamatergic input to the striatum (Plotkin and Surmeier, 2015; Reiner and Deng, 2018; V eldman and $Y$ ang, 2018). On the presynaptic side, there is a tendency for down-regulation of vGlutl immunofluorescence (Rothe et al., 2015), decrease in vGluT 1+ terminal numbers (Deng et al., 2013) and impairment of synaptic glutamate release at individual corticostriatal terminals (Dvorzhak et al., 2019). On the postsynaptic side, corticostriatal synaptic transmission might be affected by insufficient supply with brain-derived neurotrophic factor (Plotkin et al., 2014) or reduced signal transfer from distal dendrites (Carrillo-Reid et al., 2019). The duration of the NMDAR component of corticostriatal EPSCS was found to be prolonged
(Dvorzhak et al., 2019), which might be due to a reduced glutamate uptake capacity of striatal astrocytes (Tong et al., 2014; D vorzhak et al., 2016). Indeed, targeted expression of MHTT in astrocytes consistently impeded their glutamate uptake function (Shin et al., 2005;Bradford et al., 2009; Faideau et al., 2010; M eunier et al., 2016) and induced or exacerbated the motor symptoms of HD (B radford et al., 2009;M eunier et al., 2016).

In the adult rodent striatum most of the glutamate transport is carried out by the excitatory amino acid transporter type 2 (EAAT2, gene name SIcla2) (Danbolt, 2001;Beart and O'Shea, 2007; V andenberg and Ryan, 2013). The EAAT2 protein is clustered at the sites of synaptic glutamate release (Danbolt, 2001) which ensures the rapid return of the extracellular glutamate concentration to very low resting levels (Bergles and Jahr, 1998). There is general agreement that SLC1a2 expression is weaker in mouse models of HD. Moreover, spontaneous and/or pharmacologically stimulated uptake of exogenous $[3 \mathrm{H}]$-labeled L-glutamate or D-aspartate was reduced by $H D$, as shown in lysed synaptosomal preparations (Lievens et al., 2001;Bradford et al., 2009;Faideau et al., 2010; Huang et al., 2010; Parsons et al., 2016) or striatal perfusates (B ehrens et al., 2002; E strada-Sanchez et al., 2019). $N$ evertheless, a number of studies have challenged the idea that reduced expression of SIcla2 in HD astrocytes would actually translate into a state of deficient synaptic glutamate clearance (Parsons et al., 2016; Parievsky et al., 2017) or contribute to the progression of HD (Petr et al., 2013). Obviously, more information was needed to clarify the conditions resulting in the proposed mismatch between glutamate release and clearance.

In principle, a relative deficiency of glutamate uptake could result from the following distinct mechanisms: i) altered transporter-substrate interactions, ii) insufficient SIcla2 transcription, iii) impaired membrane targeting of EAAT2, and/or iv) exaggerated intracellular degradation of EAAT2. Heterologous expression studies with recombinant chimeric EAAT2 variants delineated the significance of functional compartments within the protein. It turned out that a region close to the distal end of the last transmembrane domain is relevant for the interaction with the transport substrate (Leinenweber et al., 2011), and affects the balance between cytoplasmic retention and membrane insertion (Kalandadze et al., 2002;U nderhill et al., 
2015). Due to detailed studies from the Zafra lab, it is also known that ubiquitination of $4 \mathrm{C}$-terminal lysine sites (Gonzalez-Gonzalez et al., 2008) substantially contributes to the internalization of the transporter from the plasma membrane. Finally, it was reported that sumoylated toxic C-terminal fragments may inhibit the SIcla2 transcription in the astrocyte nucleus

(Gibb et al., 2007; Foran et al., 2011;Rosenblum et al., 2017). All these studies imply that EAAT2 binding partners at the $\mathrm{C}$-terminal end participate in the regulation of glutamate uptake, but detailed information on the spectrum of EAAT2 interactors in heal th and disease is not yet available.

The present study addresses the possibility that glutamate uptake can be enhanced by the expression of genetically engineered variants of SIcla2. If so, this would validate the proposed links between EAAT2 structure, glutamate uptake activity, synaptic glutamate clearance and exploratory motor behavior.
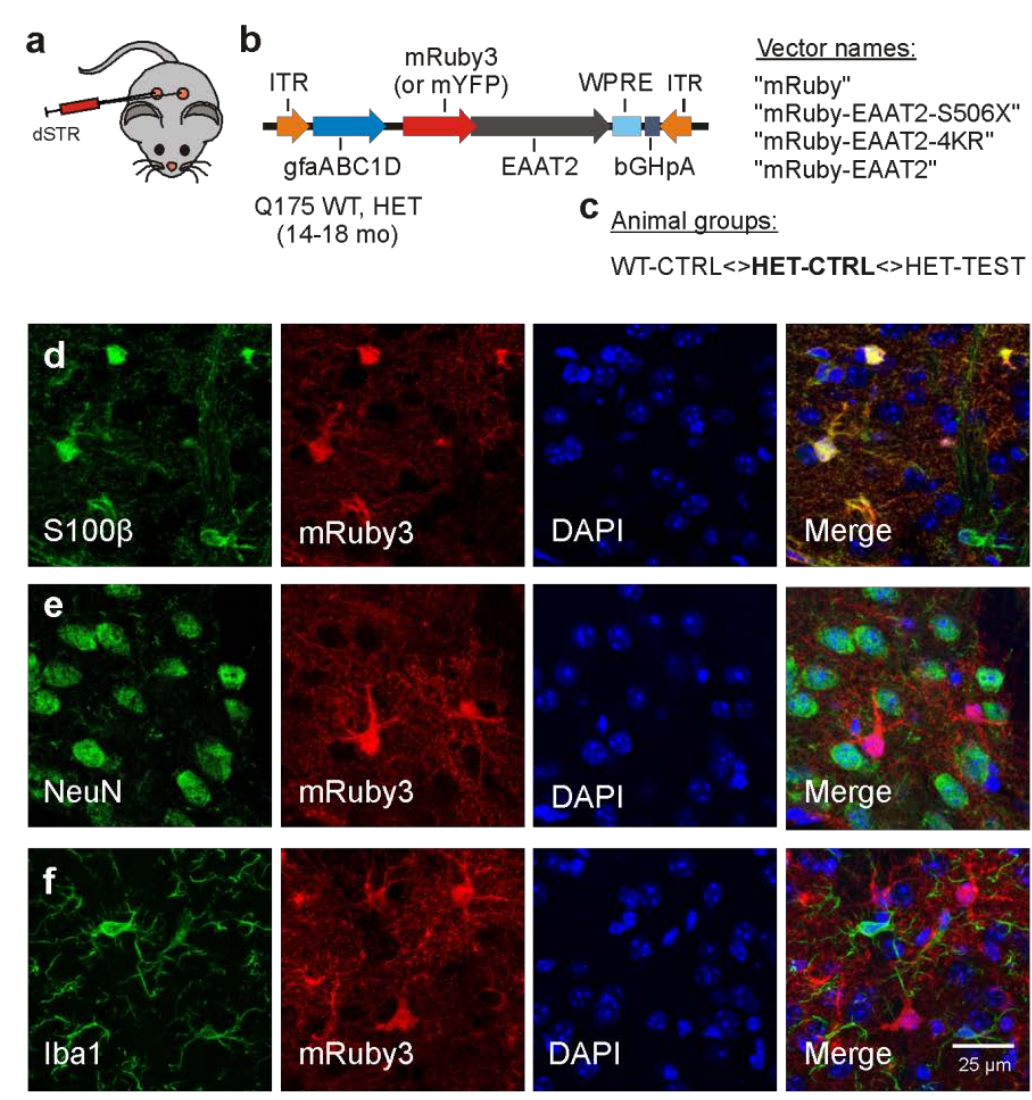

\section{RESULTS \\ Experimental approach}

Fig. 1a, $b$ illustrates the principal elements of the current series of experiments on modified SICla2 expression. All data was obtained from aged (14-18 mo) wild-type (WT) and heterozygous (HET) Q175 mice, a well established mouse model of HD (Menalled et al., 2012). A deno-associated viral vectors were used for the transfer of recombinant DNA into the dorsal striatum, the region most affected by HD-related neurodegeneration (Vonsattel and Difiglia, 1998). The respective plasmids were derived from human EAAT2, with the understanding that there is a close similarity between the human and mouse SIcla2 homologues (Kirschner et al., 1994). For detection of the transgene, mR uby3 or, in some experiments, MY FP was fused to the $\mathrm{N}$-terminus of the SIcla2 sequence. The CONTROL groups (WT-CTRL, HET-CTRL) received a vector solely encoding the fluorescent tag. The TEST groups (only HET mice)

Fig. 1. Experimental approach. $a, b$ Mouse model and types of injected vectors. $c$ General test scheme. d-f Immunostained sections for evaluation of viral expression patterns. Triple labeling with representative astrocytic (d), neuronal (e) or microglial (f) markers, together with an antibody against mRuby3 and nuclear counterstaining with DAPI. $g$ The mRuby+area at the indicated mediolateral distance. $\boldsymbol{h}$ Transduced area visualized with mRuby 3 on the background of DAPI (blue) and GFAP immunofluorescence (green). The putative margin of the striatum is marked with a dotted line. $i$ Incidence of transduced cells within the S100ß-, NeuN- or Ibal-expressing cell populations. Numbers on columns: Evaluated sections and animals (in brackets).j Fraction of the astrocytic, neuronal or microglial phenotype among the mRuby $3+$ population in the dorsal striatum. Abbreviations: dSTR - dorsal striatum, M1-CTX-primary motor cortex (M1). 
received a vector encoding one of 3 EAAT2 variants. The TEST group of HET-EAAT2-S506X expressed a strongly modified EAAT 2 variant with removal of the last 68 amino acids, i.e. almost complete truncation of the C-terminal domain. Mice belonging to the TEST group of HETEAAT2-4KR expressed EAAT2 with four point mutations at the $\mathrm{C}$-terminal domain. The mice of the third TEST group expressed the full-length EAAT2. Thus, the 3 TEST groups represented three degrees of C-terminal modification, from almost complete truncation to full-length EAAT2. HET$C T R L$ is the principal reference group for comparison with WT-CTRL (to characterize the HD-phenotype) and HET-TEST (to determine the effect of treatment).

The expression of the transgene was limited by the chosen experimental conditions, including i) the amount of injected particles (a single injection of $1.0^{*} 10^{9} \mathrm{gc} /$ striatum), ii) the expression time (3-5 weeks, if not mentioned otherwise), iii) the type of the viral serotype (PHP.eB) and iv) the promoter sequence (gfaABC1D). To examine the abundance and specificity of transduction we analyzed immunostained parasagittal sections from fixed brains (Fig. 1d-f). As functional imaging experiments were limited to cells in the dorsal striatum, counts were performed at a mediolateral distance of $2-2.5 \mathrm{~mm}$ within the boxed area. Fig. $1 \mathrm{~g}, \mathrm{~h}$ illustrates that the area of evaluation was always well within the extensively labeled zone. The latter covered about $40 \%$ of the striatal volume. An "effective transduction rate" (ETR) was determined in 4 mice ( $2 \mathrm{WT}$ and $2 \mathrm{HET}$ ), each contributing about 10 viewfields. Co-localization of mRuby3 with a suitable cell-specific marker rendered the following ETR values (Fig. 1i): S100 $\beta+$ cells (astrocytes) $-65.9 \pm 2.47 \%$, N euN + cells (neurons) - $2.76 \pm 0.44 \%$, Ibal+ cells (microglia) $-1.64 \pm 0.85 \%$. There also was some labeling in the overlaying cortex and a small degree of unintended transgene expression in neurons and microglial cells. A mong the population of mRuby+ cells in the dorsal striatum (boxed area in Fig. 1h), $90.8 \%$ were astrocytes, $8.3 \%$ - neurons and $1.6 \%$ microglial cells (Fig. 1j).

The cell counts indicate that the viral transduction was local (dorsal striatum), specific (astrocytes) and effective (two thirds of the astrocytes in the labeled area). This expression model was therefore considered suitable to characterize the HD phenotype, on one side, and the response to the exogenous EAAT 2 variants, on the other side.

\section{M otor performance of control and treated HD mice}

HET mice exhibit the symptoms of hypokinesia at an age of 10-12 months meaning that at the time of injection and testing neurons and astrocytes in the striatum and elsewhere have undergone changes for several months. The aim of the following experiments was to quantify the HD-induced changes in locomotion and to find out to what extent these changes could still be reversed. To gain a set of easy-to-quantify indicators of hypokinesia the mice were submitted to two video-recorded tests: i) the step-over latency test, SO LT (Dvorzhak et al., 2019) and ii) the open field test, OFT (M enalled et al., 2012; Rothe et al., 2015). The tests were performed immediately before the viral vector injection and 3-5 weeks later, before animal sacrifice. This behavioural examination provided us with a set of 6 criteria to classify an animal or animal group as being hypokinetic or recovered from hypokinesia.

Specifically, the step-over latency of SOLT was regarded as a direct measure of the time needed to initiate exploration in the open field. Its usefulness was supported by a larger number of observations from non-injected HD mice (Supplemental Fig. 1). Step-over latencies longer than $300 \mathrm{~ms}$ were almost exclusively found in HET which could contribute to the detection of recovery effects. The other indicators are based on a more complex analysis of the movement trajectories in the open field (Fig. 2a, $b$, see Supplemental text for definitions of the analysed parameters). In the present experiments with the injected WT-CTRL, HET-CTRL and HET-EAAT2-S506X TEST mice, 5 out of 6 movement parameters extracted from OFT were sensitive to the $\mathrm{C}$-terminal-modified EAAT2. The most commonly used OFT indicator "total distance travelled in $5 \mathrm{~min}$ " was found to be decreased in the HET-CTRL group and increased in HET-S506X if compared to HET-CTRL (Fig. 2a, c). The incidence of starts from rest is possibly the most reliable indicator of depressed locomotion (Fig. 2a, d). It is inversely correlated with the step-over latency (Fig. 2f). After treatment with mYFP-EAAT2-S506X both parameters exhibited changes towards WT levels (Fig. 2d, f). A Iso noteworthy is the treatmentrelated reduction of the maximal to mean radius of the resting area, i.e. the extension of the resting area until a new movement starts (Fig. 2b, g). O ne could 


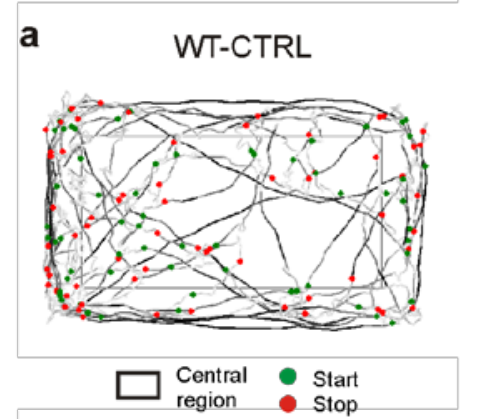

HET-CTRL

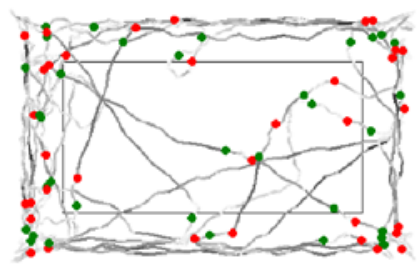

$100 \mathrm{~mm}$

b
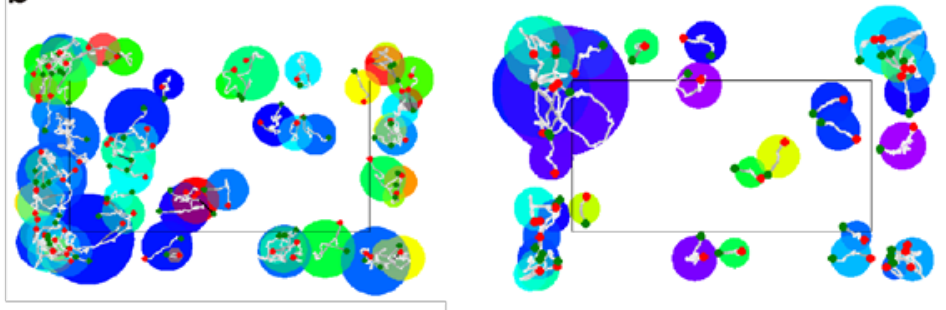

HET-EAAT2-S506X
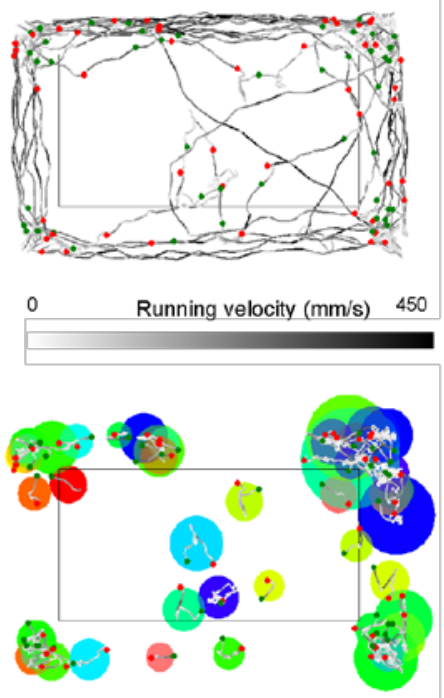

$0 \quad$ Velocity at rest $(\mathrm{mm} / \mathrm{s}) \quad 150$

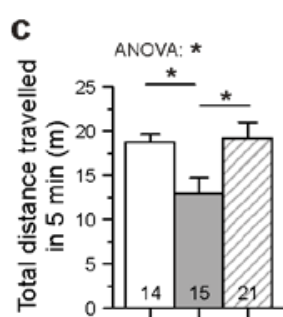

से से<smiles>c1ccc2c(c1)CCCC21CCCCC1</smiles>

h

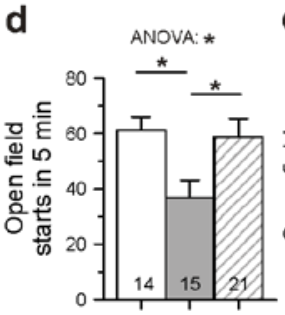

Pearson: $* \star$
$r .-0.3925(n=49, p=0.0053)$

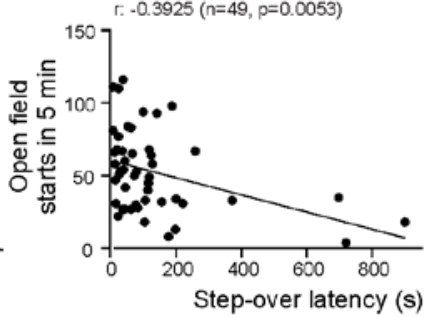

f

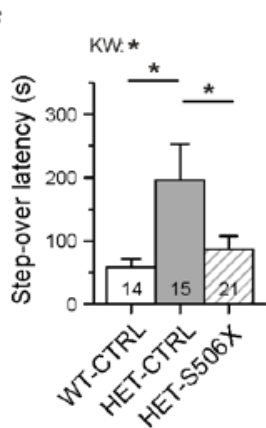

$\mathbf{k}$
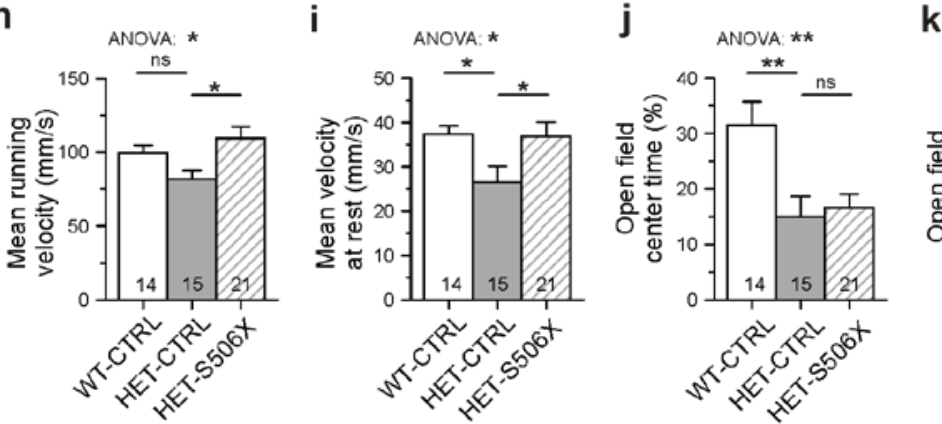

i

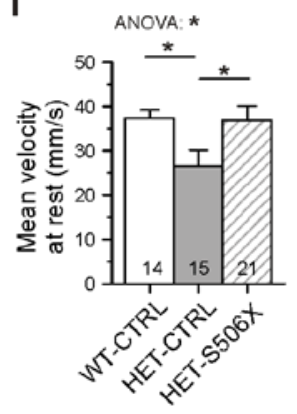

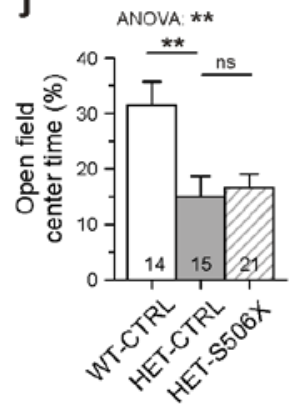

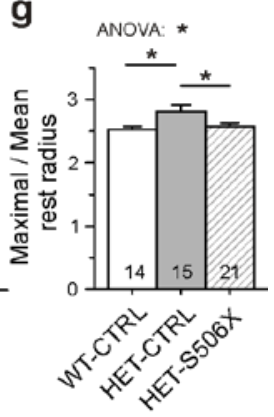

I

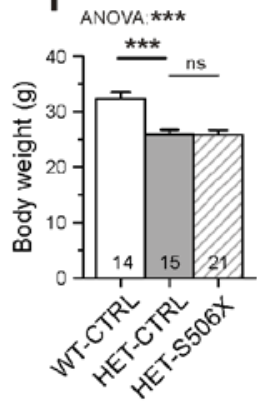

Fig. 2. Effects of bilateral EAAT2-S506X expression on the motor performance of Q175 mice. a Recordings of open field trajectories showing the mean ruming velocity between starts and stops (green or red circles, respectively). Gray level coding of instantaneous running velocity. $\boldsymbol{b}$ Motor activity at rest. Color coding of movement velocity at rest. $\boldsymbol{c}, \boldsymbol{d}, \boldsymbol{f}$ - $\boldsymbol{k}$ Quantification of results from open field testing. $e$, f Inverse relationship between the number of starts from rest and step-over latency, and sensitivity of the latter to HD and treatment. I matched age-, CAG-and body-weight composition of the test groups (only body weightillustrated).

further expect that HD or treatment can alter the velocity of body displacement, but it is not known whether this would equally apply to rest and running. Previous open field experiments with simultaneous recording of motor activity and local field potentials (LFPs) suggested that the HDrelated synchronisation of striatal activity only occurs during the phases of rest thereby underlining the distinct character of the resting condition (R othe et al., 2015). Now it turned out, that movement velocity is affected both during the resting and running phases. Moreover, the direction of the changes induced by HD and treatment were the same (Fig. 2h, i). Other aspects of open field behavior were unchanged by viral treatment, such as the relative time spent in the open field center, or the open field resting time (Fig. 2j, k) which suggests that these indicators might be unrelated to 
bioRxiv preprint doi: https://doi.org/10.1101/2020.09.17.302158; this version posted March 26, 2021. The copyright holder for this preprint (which was not certified by peer review) is the author/funder. All rights reserved. No reuse allowed without permission.

the site of injection, the dorsal striatum, or not be representative of exploratory motor activity.

A mong the less effective attempts to return the functional indicators of HET to WT performance were experiments with mice expressing a fulllength EAAT2 variant. In this case the enhancement of locomotion was small, if at all present (compare the corresponding graphs in Fig. $2 \mathrm{c}-\mathrm{i}$ and Supplemental Fig. 2a-e). The statistical analysis showed that EAAT 2-S506X-treated mice outperformed not only with respect to HET-CTRL but also with respect to HET-EAAT2. This was significant (ANOVA $p<0.05$ ) for the number of open field starts stops in $5 \mathrm{~min}$ and the mean running velocity $(\mathrm{mm} / \mathrm{s})$. The respective mean, $\mathrm{SE}$ and $\mathrm{N}$ were for HET-CTRL: $36.80 \pm 6.23$ and 81.57 $\pm 6.04(\mathrm{~N}=15)$, HET-EAAT2-S506X: $58.67 \pm 6.66$ and $109.45 \pm 8.06(\mathrm{~N}=21), \mathrm{HET}-\mathrm{EAAT} 2: 41.40 \pm$ 7.38 and $94.45 \pm 8.57(\mathrm{~N}=10)$.

Together, these experiments suggest i) that the state of striatal astrocytes is relevant for the incidence and speed of exploratory movements, ii) truncation of the EAAT2 C-terminal might be an effective means to reduce locomotor deficits in HD mice and iii) induced over-expression of full-length $\mathrm{SICla}_{2}$ is not sufficient to alleviate the symptoms of hypokinesia.

\section{Astrocytic glutamate uptake}

To gain further information on the functional consequences of EAAT2 modifications in HD, imaging experiments were performed in striatal slices loaded with the $\mathrm{Na}^{+}$indicator SBFI-AM. Glutamate transport was elicited with $L$-aspartate. It is known that $\mathrm{L}$-glutamate, $\mathrm{L}$-aspartate $\mathrm{D}$-aspartate are taken up with similar micromolar affinity (A rkhipova et al., 2019). In the present experiments $\mathrm{L}$-aspartate was chosen for its physiological role as excitatory neurotransmitter (M orland et al., 2013) and its negligible effects at G-protein-coupled glutamate receptors. The major part of the $L$ aspartate-induced response was blocked by the a
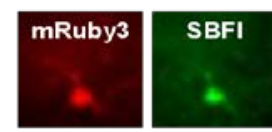

L-Asp $1 \mathrm{mM}$
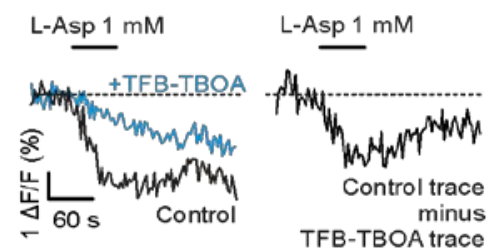

b

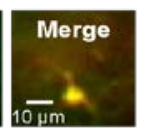

TFB-TBOA trace

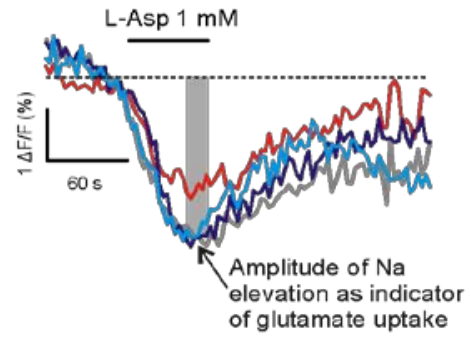

c

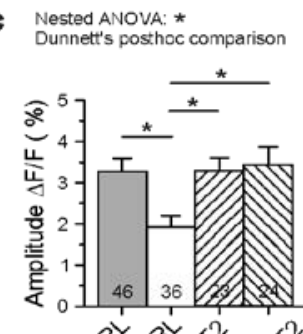

e WT-CTRL HET-CTRL HET-EAAT2-S506X
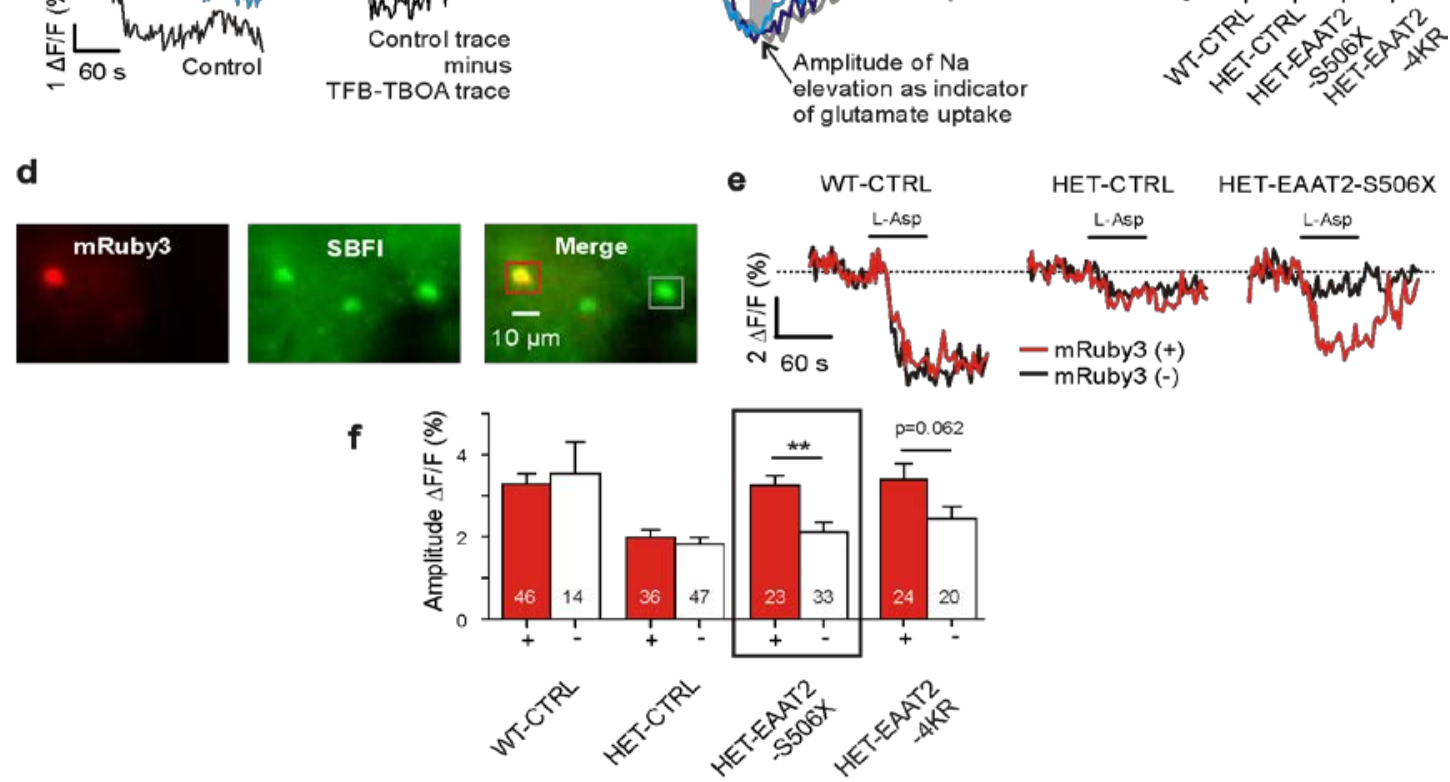

Fig. 3. Rescue of glutamate uptake activity of striatal astrocytes after bilateral expression of EAAT2-S506X or EAAT2-4KR a Measurement of glutamate uptake by sodium imaging with SBFI. Traces recorded in the absence and presence of TFB-TBOA $(2$ $\mu M$ ). The amplitude of this differential response was expressed as $\Delta F / F$ during the last 15 sec of $L$-Asp application. F is the mean fluorescence at rest, before drug application. b Averaged traces of SBFI fluorescence. Results from mRuby3-positive astrocytes only. $c$ Quantification of the L-aspartate-induced sodium elevation. Two-level ("nested") statistics (animal level, cell level and posthoc comparison between groups). d The applied SBFI loading protocol was selective for astrocytes. Viewfield with 3 astrocytes, where one is transduced and two are not. e Traces from mRuby+ as opposed to mRuby-in three test groups. Averaged traces from one animal per group. f Comparison of results obtained in the different groups from mRuby3+vs. mRuby3-astrocytes. 
high-affinity glutamate transport inhibitor TFBTBOA (Fig. 3a), and this TFB-TBOA-sensitive component of the $\mathrm{Na}^{+}$elevation was quantified in WT-CTRL, HET-CTRL and two HET-TEST groups, HET-EAAT2-S506S and HET-EAAT2$4 K R$. The recordings of averaged responses (Fig. $3 b)$ visualize the differences between $W T$ and $H E T$ and the similarity of responses obtained in the WTCTRL, EAAT2-S506X and EAAT2-4KR groups. Multilevel (nested) ANOVA confirmed a significant rescue of the $L$-aspartate-induced sodium response in astrocytes transduced with EAAT2-S506X and EAAT2-4KR (Fig. $3 \mathrm{C}$ and Supplemental Tab. 1). The present SBFI-loading protocol was adjusted to preferentially label astrocytes (Dvorzhak et al., 2016). This offered an opportunity to determine, in the same viewfield, the $\mathrm{L}$-aspartate-induced glutamate uptake activity of transduced versus non-transduced astrocytes (Fig. $3 d, e)$. In the case of HET-EAAT2-S506X, there was a significant difference between the transduced and not-transduced astrocytes (Fig. 3f).

SBFI imaging was also performed in mice injected with the vector encoding full-length EAAT2 (Supplemental Fig. 2f, g), but significant rescue of glutamate uptake was not observed. Statistical analysis of the $\mathrm{L}$-aspartate-induced $\mathrm{Na}$ transients in HET-CTRL, HET-EAAT2-S506X and HETEAAT2 (Kruskal-Wallis test: $P=0.0004$ ) showed that EAAT2-S506X -treated mice outperformed not only with respect to HET-CTRL (Dunn's test: $P=$ 0.0004 ) but also with respect to HET-EAAT2 (Dunn's test: $P=0.0052$ ). The respective mean, SE and $N$ of $\triangle F / F(\%)$ were for HET-CTRL: $2.01 \pm$ $0.18(\mathrm{~N}=36)$, HET-EAAT2- S506X : $3.28 \pm 0.23$ ( N $=23)$, HET - EAAT 2: $2.31 \pm 0.23(\mathrm{~N}=34)$.

These results show that the expression of the truncated but not the full-length EAAT2 variant effectively counteracted the HD-induced depression of glutamate uptake.

\section{Possible effects on neuronal excitability and results of systemic vector application}

A series of supplemental experiments addressed the influence of HD and EAAT2-S506X treatment on neuronal excitability (Supplemental Fig. 3) and the efficacy of treatment with systemically applied vectors (Supplemental Fig. 4). In both cases the effects of S506X remained below the significance level required with nested ANOVA. These experiments may however provide useful information on the HD-related pathologies in striatal astrocytes of aged mice.

\section{G lutamate clearance at corticostriatal synapses}

The significance of astrocytic glutamate uptake deficits for glutamatergic synaptic transmission in the striatum is still a matter of debate. A previous publication from our lab (Dvorzhak et al., 2019) has addressed this controversy by using CaM K IIdriven expression of the genetically encoded

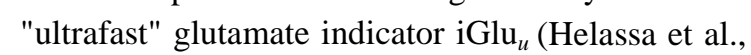
2018) to examine the performance of single corticostriatal synapses in WT and Q175 HET. It was found that in HET aged 15 to 19 months at least $40 \%$ of corticostriatal synapses exhibited a deficit in the glutamate clearance. A rescue of the synaptic glutamate clearance function in symptomatic HD mice after a molecular intervention targeting the astrocytic glutamate uptake would be a strong argument in support of the clearance-deficit hypothesis of HD. We therefore examined the effect of the truncated EAAT2-S506X transgene expression on the properties of single corticostriatal synapses in a group of HD mice with manifest symptoms of hypokinesia.

Large $(>0.63 \mu \mathrm{m})$ fluorescent terminals were assumed to belong to the group of PT-type afferents formed by the layer $V$ pyramidal neurons of the motor cortex (Reiner and Deng, 2018; D vorzhak et al., 2019). The specimen recordings selected for Fig. 4a illustrate the differential spread and duration of the glutamate el evation in the perisynaptic space. Only terminals located on the territory of transduced astrocytes were included in the test samples. The arrowhead of Fig. $4 \mathrm{~b}$ points to a bright varicosity on the background of the red fluorescent area generated by the dendritic field of one mRuby-expressing astrocyte. These spatial interrelations are relevant for a better understanding of synaptic modulation by astrocytic transporters or gliotransmitters.

It can be seen that the parameter "Spread" (see $M$ ethods for definition) was sensitive to treatment with EAAT2-S506X (Fig. 4a, d). Fig. 4c shows the time-dependent changes of the $\mathrm{iGlu}_{u}$ intensity values before and after a short biphasic electrical stimulus applied through a glass pipette to the iGluu-expressing presynaptic terminal. Such traces could be used to extract two important indicators: "Peak amplitude" (short red horizontal line) and "Time constant of glutamate concentration decay" 

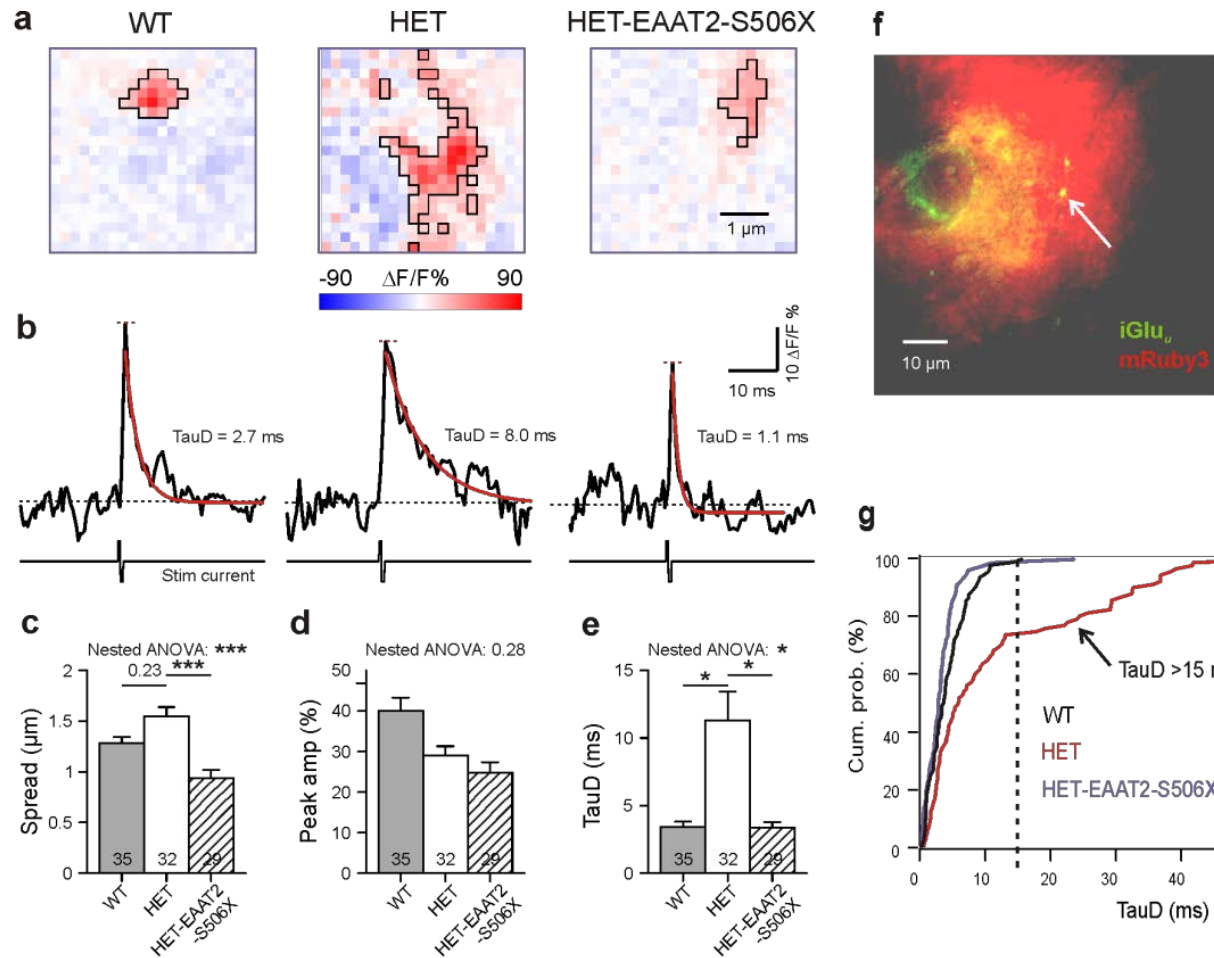

d
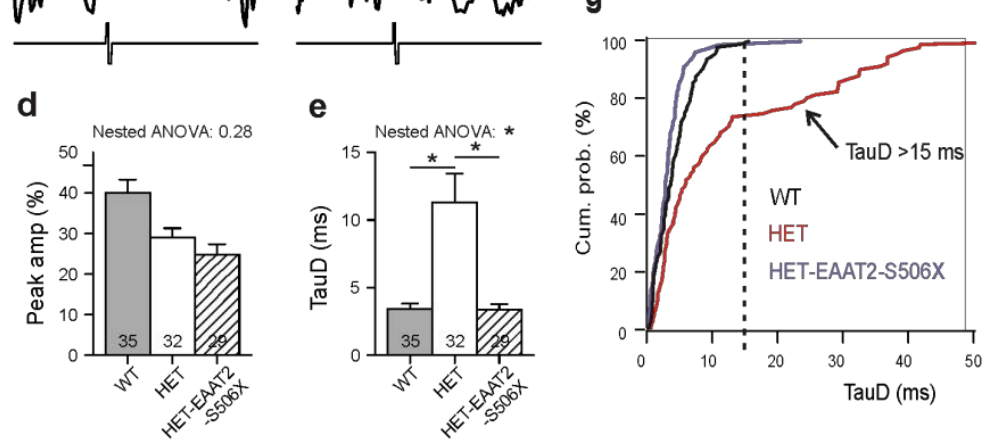

Fig. 4. Rescue of single synapse glutamate clearance in the striatum after expression of EAAT2-S506X. a Specimens from single PT-type corticostriatal synaptic terminals in slices from WT, HET and treated HET. Bilateral intracranial injections of HET with EAAT2-S506X. The selected images were acquired at the response peak to illustrate the extension of $i$ Glu elevation (boxed area). Pink pixels within the black boundaries are pixels where stimulation of the bouton elicited a fluorescence increase $(\Delta F)$ to values larger than the resting level prior to stimulation $(F)$. $b$ Corresponding $i G l u_{u}$ values before and after electrical stimulation of the given bouton in the presence of TTX. The traces represent the mean value calculated from all suprathreshold pixels in the ROI. Red curves: Monoexponential functions fitted to the iGlu traces. In black - respective time constants of decay (TauD). c-e Quantification of results. $f$ Only synapses in the immediate vicinity of transduced astrocytes were included, as confirmed by the location of the tested $i G l u_{u}$-expressing varicosity on the territory of mRuby3-positive astrocytes (arrowhead). $\mathrm{g}$ Cumulative probability plot to illustrate the absence of TauD values $>15 \mathrm{~ms}$ in WT and in EAAT2-S506X-treated HET. TauD values $>15 \mathrm{~ms}$ identify pathological synapses in HET.

( $T$ auD, see values next to the red monoexponential fitting curve). Similar traces were also constructed from single pixels. The transients derived from the pixel with the highest fluorescence increase reflect the release and clearance of glutamate next to the presynaptic active zone, i. e. the site of vesicle exocytosis. The respective results are given in the Supplemental Tab. 1.

The data presented in Fig. $4 \mathrm{~d}-\mathrm{f}$ is based on the mean suprathreshold pixel intensity values. It can be seen that Spread and TauD, but not Peak amplitude, were affected by the expression of EAAT2-S506X. It is, thus, very likely that Spread and TauD reflect the astrocytic glutamate uptake while the Peak amplitude indicates the amount of released glutamate (Dvorzhak et al., 2019). TauD values larger than $15 \mathrm{~ms}$ have never been observed in WT while more than one third of the HET synapses tested at an age of $\sim 16$ months showed TauD values $>15 \mathrm{~ms}$. Now we found that in HET treated with EAAT2-S506X synapses with prolonged decay were entirely absent (Fig. $4 \mathrm{~g}$ ).

These results demonstrate i) a contribution of the astrocytic glutamate uptake deficit to the HDrelated pathology in the corticostriatal pathway and ii) a return of synaptic glutamate clearance towards wild-type levels after expression of the $\mathrm{C}$-terminaltruncated EAAT 2 variant.

\section{Native intrinsic EAAT2 in immunostained corticostriatal synapses}


bioRxiv preprint doi: https://doi.org/10.1101/2020.09.17.302158; this version posted March 26, 2021. The copyright holder for this preprint (which was not certified by peer review) is the author/funder. All rights reserved. No reuse allowed without permission.

Next it was asked whether effects of EAAT2S506X (or EAAT2-4K R) could also be discerned in EAAT2-immunostained sections. It should be noted that the selected antibody $(A B)$ only reacts against
EAAT2-4KR groups differed from the HET-CTRL group, as their synaptic EAAT2 protein level increased to nearly WT level.
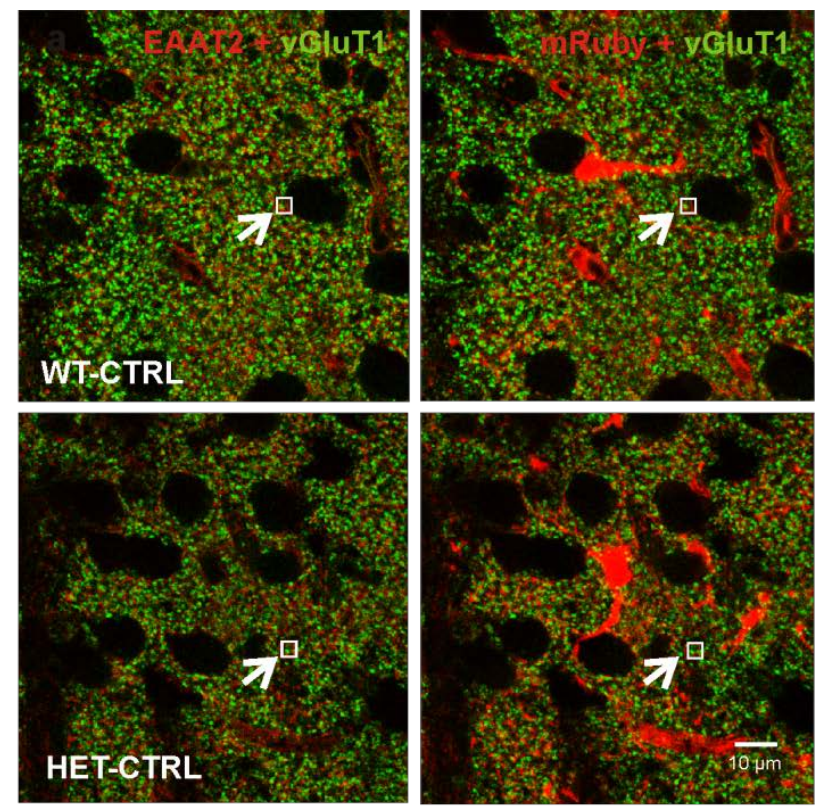

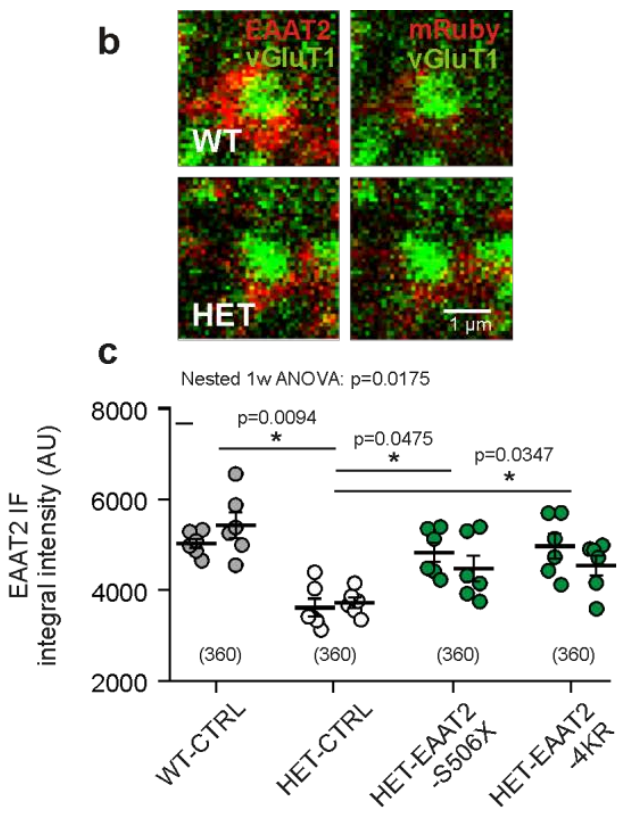

Fig. 5. Evaluation of synaptic EAAT2 immunofluorescence in fixed sections. a Confocal images from WT-CTRL and HETCTRL to illustrate the HD-related decrease in the overall EAAT2IF. In the illustrated samples the mean intensity values were 3078 \pm 38.4 (WT-CTRL) and $2673 \pm 31.7$ (HET-CTRL). b Regions of interest (ROIs) comprising just one synapse. The ROIs correspond to the small boxed areas in $a$. The EAAT2-immunopositive area corresponds to an astrocyte PAP, as it is labeled with mRuby3. $c$ Quantification of integral EAAT2 IF from ROIs comprising only one vGluT1-immunopositive terminal. Numbers in brackets:

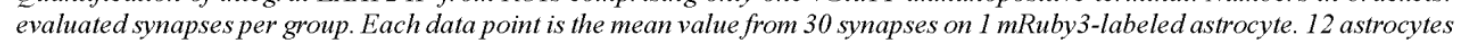
per group from 2 mice/group. Statistics: Nested data analysis.

EAAT2 with intact C-terminal. This provided us with an opportunity to examine the effect of transgene expression on the native perisynaptic EAAT2. Fig. 5 shows the results from four simultaneously processed test groups, each consisting of two CAG-, age- and body weightmatched animals: WT-CTRL, HET-CTRL, HETEAAT2-S506X and HET-EAAT2-4KR. The images obtained from the immunostained sections (Fig. 4a) illustrate, first of all, a noticeable shift towards lower EAAT2 intensity levels in HET, thereby confirming previous studies from noninjected HD mice (Estrada-Sanchez et al., 2009; Tong et al., 2014). Fig. 5b shows the respective single synapse immunofluorescence (IF) in ROIs containing only one vGluT 1-positive spot, i.e. one corticostriatal presynaptic terminal. The EAAT2-positive pixels co-localized with mRuby3, i.e. they belongrd to an astrocyte process. The graph of Fig. $5 c$ and the Supplemental Tab. 1 show the results of statistical evaluation of the EAAT2immunofluorescent clusters within the analyzed ROIs. Both the HET-EAAT2-S506X and the HET-
The data from immunostained brain sections suggest an effect of the $\mathrm{C}$-terminal-modified EAAT2 on the level of the native murine EAAT2 at corticostriatal synapses.

\section{W estern blot (W B) analysis of native EAAT 2}

The following experiments were designed to further examine the possibility that expression of a $\mathrm{C}$ terminal-modified EAAT2 transgene in the HET striatum can increase the amount of native EAAT2. A bout 15 months old Q175 WT and HET were sacrificed 3-4 weeks after viral injection to prepare striatal lysates for WB analysis. The lysates contained both the "foreign" YFP-tagged EAAT2 transgenes and the "intrinsic" native EAAT2 protein without tag (Fig. 6a). The compared animal groups are listed in Fig. 6b. An overview of all possible WB bands is given in Fig. $6 c$. The native EAAT2 monomers and dimers are represented in the $70 \mathrm{kDa}$ and $140 \mathrm{kDa}$ bands, respectively. Due to the $C$-terminal binding characteristics of the EAAT2 Ab, immunoreactivity (IR) is only observed at the native EAAT2 and YFP-EAAT2 
bioRxiv preprint doi: https://doi.org/10.1101/2020.09.17.302158; this version posted March 26, 2021. The copyright holder for this preprint (which was not certified by peer review) is the author/funder. All rights reserved. No reuse allowed without permission.

bands but not the C-terminal truncated YFPEAAT2-S506X band (compare boxed area in Fig. $6 \mathrm{~d}$ and e). Evaluation of the striatal lysates from HET-EAAT2-CTRL and HET-EAAT2-S506X showed that the amount of native EAAT2 was indeed higher in the HET-EAAT2-S506X group (Fig. 6f) which lends further support to the suggestion that the transgene and native EAAT2 proteins interact.
Together with the data in Fig. 5, these findings provide a first hint on the mechanism underlying the behavioral and cellular rescue effects in HD mice treated with the truncated EAAT2 transgene. The results are consistent with the idea that expression of the S506X variant can increase the availability of native EAAT2.

\section{Proteomics analysis of the EAAT2 interactor spectrum in the striatal immunoprecipitate}

a

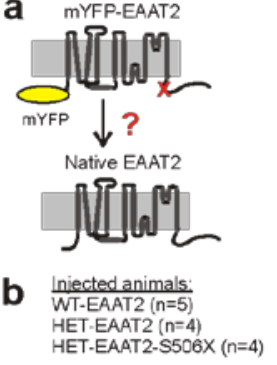

MYFP-EAAT2

C

\begin{tabular}{|c|c|l|c|c||c|c|}
\hline Lysate & Beads & Protein & & MW & WB & Symbol \\
\hline $\mathrm{x}$ & & Native EAAT2 monomer & 62 & $\sim 70$ & $\mathrm{E}$ \\
\hline $\mathrm{x}$ & & Native EAAT2 dimer & 124 & $\sim 140$ & $\mathrm{E}-\mathrm{E}$ \\
\hline $\mathrm{x}$ & $\mathrm{x}$ & MYFP-EAAT2 monomer & 89 & $\sim 100$ & YE \\
\hline & $\mathrm{x}$ & mYFP-EAAT2 dimer & 178 & $\sim 200$ & YE-YE \\
\hline & $\mathrm{x}$ & mYFP-EAAT2 + native EAAT2 & 151 & $\sim 170$ & YE-E \\
\hline $\mathrm{x}$ & $\mathrm{x}$ & mYFP-EAAT2-S506X & 81 & $\sim 90$ & YEX \\
\hline & $\mathrm{x}$ & mYFP-EAAT2-S506X dimer & 162 & $\sim 180$ & YEX-YEX \\
\hline & $\mathrm{x}$ & mYFP-EAAT2-S506X + native EA/ & 142 & 143 & $\sim 160$ & YEX-E \\
\hline
\end{tabular}

$$
\text { d }
$$

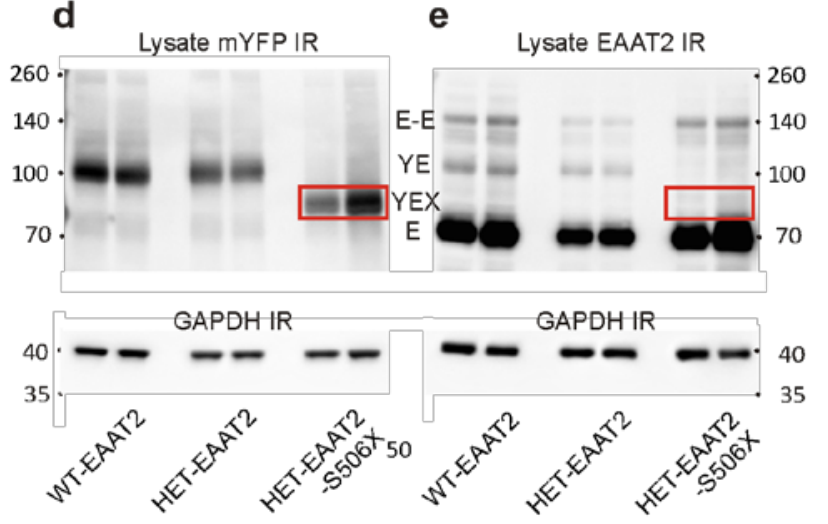

G

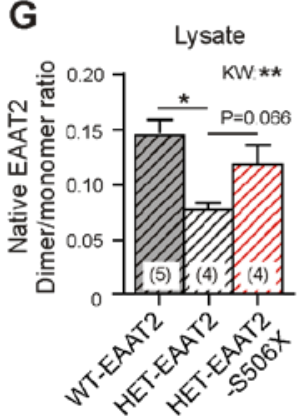

f

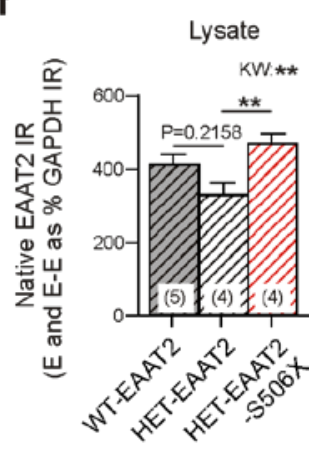

h

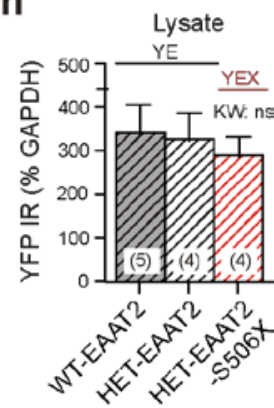

Fig. 6. Western blot (WB) analysis to examine the changes in native murine EAAT2. a Two principal forms of EAAT2 to be detected in the lysates: Native murine EAAT2 and recombinant YFP-EAAT2 expressed due to virus injection. $b$ Test groups according to injectedmice. $c$ EAAT2 bands to be detected in the WB. Info for the immunoprecipitate (beads) to be used for Fig. $7 b$. d, e WB samples prepared for the quantification of YFP and EAAT2 IR in striatal lysates. At least four animals per group (shown are two). The boxed band YEX is not detected in e since the preferred antibody against EAAT2 binds to a region located on the deleted C-terminal. fMean intensity of native EAAT2 IR (mono- and dimers). Note significantly higher amount of native EAAT2 in HETs expressing the truncated as opposed to full-length EAAT2 variant. $g$ Quantification of the dimer to monomer ratio E-E/E. $\boldsymbol{h}$ Similar amounts of $m$ YFP IR (YE or YEX bands) expressed in the 3 animal groups. Nonparametric Kruskal-Wallis test (KW) and Dunn's posthoc comparison applied in all 3 graphs.

It is conceivable that the smaller amounts of synaptic EAAT2 in HD were associated with a deficit in multimer formation. Under the given experimental conditions, i.e. W estern blotting, only a small percentage of EAAT2 (less than 15\%) retained or re-established a dimeric form. Nevertheless, it could be shown that the dimer/monomer ratio of native EAAT2 was lower than in the HET-EAAT2 as compared to the WTEAAT2 group. But the treatment effect remained below significance level. Of note, the amount of the mYFP-tagged transgene was not different between the test groups (Fig. 6h).
Further understanding of the signaling pathways underlying the regulation of astrocytic glutamate transport would require a better knowledge of the involved EAAT2 binding partners. Such information could be obtained from massspectrometry-based analysis of the EAAT2 interaction proteome after a "pull-down" of YFPtagged EAAT2. Fig. 7a illustrates the selected method of immunoprecipitation from striatal lysates where nanobody-coated magnetic beads served as "bait" for the YFP fused to the EAAT2 protein. The animal groups were the same as in Fig. 6: WT-EAAT2, HET-EAAT2 and HET-EAAT2S506X. The quality of the immunoprecipitate was 
bioRxiv preprint doi: https://doi.org/10.1101/2020.09.17.302158; this version posted March 26, 2021. The copyright holder for this preprint (which was not certified by peer review) is the author/funder. All rights reserved. No reuse allowed without permission.

verified by $W B$ analysis of YFP- and EAAT2-IR (Fig. 7b, C). The total amount of YFP pulled down by the beads was very similar in the 3 tested groups (Fig. 7d), as should be the case if the amounts of "bait" were equal in the 3 groups, and the amount of "prey" (YFP-EAAT2) would have sufficed to saturate the binding sites.

The immunoprecipitate was then submitted to arranged according to the size/direction of the HDrelated differences. Significant differences in the abundance of a given interactor are denoted by asterisks. The empty bars/black asterisks reflect the differences between the WT-EAAT2 and HETEAAT 2 groups, i.e. the effects of HD as such. The filled bars/green asterisks show the respective log2 differences between the HET-EAAT2-S506X and the HET-EAAT 2 groups. This data illuminates the
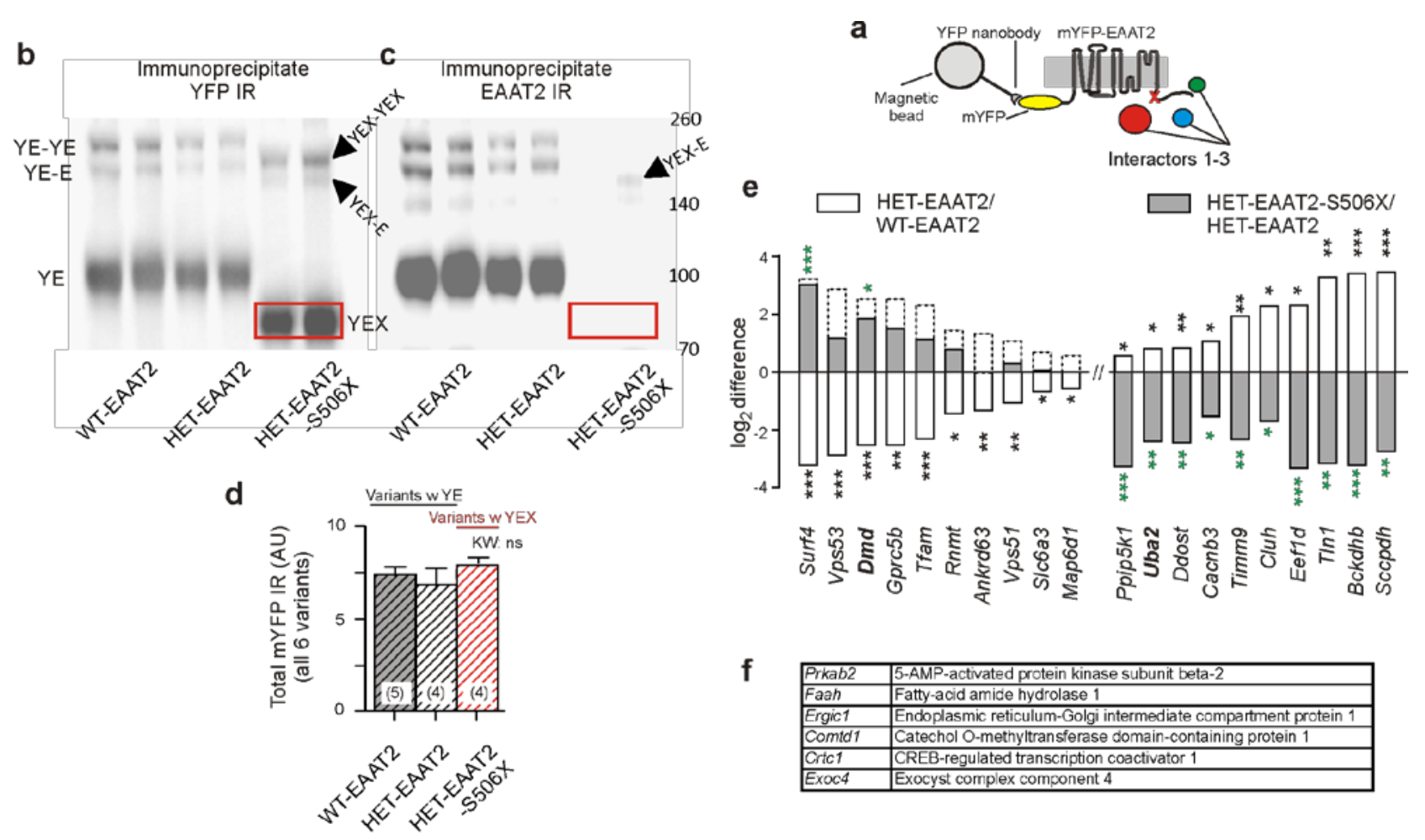

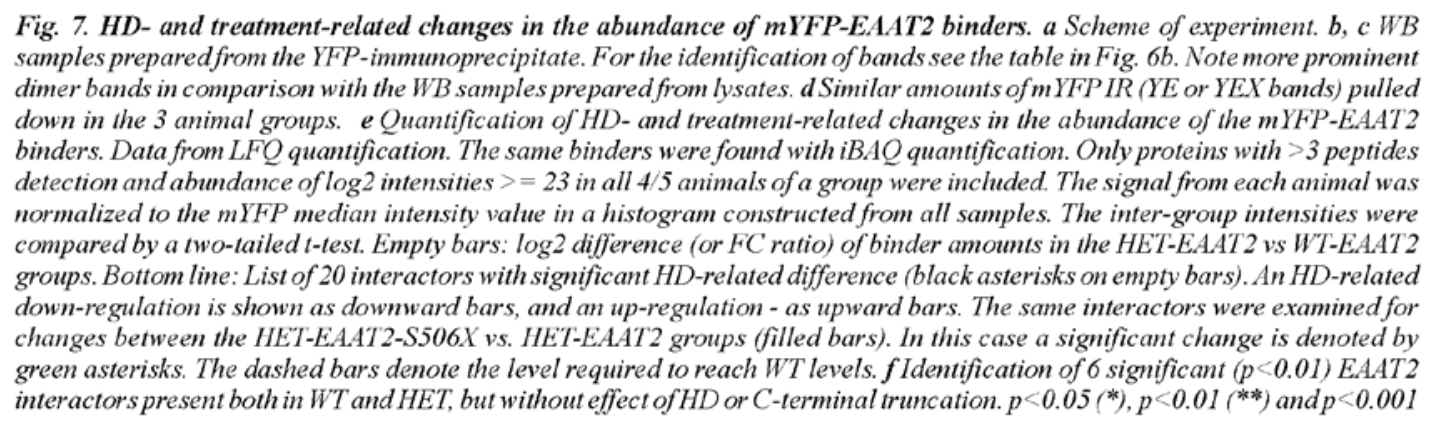

liquid chromatography tandem mass spectrometry (LC-MS) for further analysis of the EAAT2 Cterminal binder spectrum (Fig. 7a). The following experiments were designed to compare i) the amount of full-length EAAT2 interactors in HET vs. WT and ii) the amount of truncated vs. fulllength EAAT2 interactors in HET. Each experiment was performed in at least 4 mice, with 2 repetitions per animal. Results not confirmed by a 2 nd run were discarded. Fig. 7e lists 20 EAAT 2 interactors impact of $\mathrm{C}$-terminal truncation and may include rescue effects. Please note that the experiments cannot show what actually happens to the native murine EAAT2 protein devoid of $\mathrm{mYFP}$, but rather provide a first glimpse at the normal and pathological EAAT2 binding partners that may influence the glutamate uptake function in striatal astrocytes of intact mice. It can, for instance, be seen that the interaction with dystrophin (gene name $D m d$ ) is decreased in HD and partially 
restored in HET treated with EAAT2-S506X. M ost interesting, HD is also associated with a substantial up-regulation of potential EAAT2 interactors, an example being UBA2, the ubiquitin-like 1activating enzyme E1B, also known as SUMOactivating enzyme subunit 2 with the gene name Uba2. This interaction is strongly reduced by the truncation of the C-terminus in the HET-S506X group.

Other highly abundant or significant interactors of EAAT2 were not affected by HD (Fig. 7f) or not covered by the test. The present assay with a unique peptide number of $>3$ included 3.790 from a total of 13.000 proteins so far identified in the mouse brain (Sharma et al., 2015).

These proteomics results are consistent with the hypothesis that: i) the EAAT2 interactome is influenced by $H D$, ii) some of the HD-related changes are reversible and iii) expression of $\mathrm{C}$ terminal-truncated EAAT2 may block abnormal interaction in favor of more physiological protein interaction patterns.

\section{DISCUSSION}

The results obtained with intrastriatal injection of viral EAAT2 constructs support the hypothesis that HD-related deficits can be alleviated by the expression of a C-terminal-modified EAAT2 transgene. This conclusion is based on changes in self-induced locomotion, astrocytic glutamate uptake, synaptic glutamate clearance and synaptic EAAT2 protein levels. Together, the results underscore the importance of $\mathrm{C}$-terminal-dependent EA A T 2 binding partners in HD-related pathologies.

\section{Is region- and cell-type-restricted expression of artificial EAAT 2 variants a useful approach to study disease-related pathology?}

$H D$ is a severe inherited neurological disorder with diverse clinical symptoms and variable onset. With a respective gene test it can be diagnosed much before the onset of motor or other symptoms. Great efforts are therefore being made to reduce the expression of $\mathrm{mHTT}$ inside and outside the central nervous system before the disease actually starts (M rzljak and Munoz-Sanjuan, 2015;Tabrizi et al., 2019). MHTT-lowering therapies have reached an advanced stage of preclinical testing or even entered the phase of first clinical trials (Mullard, 2019). In animal models of HD, the most effective approaches include the use of self-inactivating $\mathrm{K}$ amiCas9 system for editing the huntingtin gene
(Merienne et al., 2017), injection of mHTT transcription-targeting viral vectors (Zeitler et al., 2019) and application of siRNAs, shRNAs or miRNAs for the reduction of mHTT mRNA and protein level (see (K aemmerer and Grondin, 2019) for review).

It is obvious that gene therapy is becoming more problematic at symptomatic stages of the disease. In $H D$, as well as other neurodegenerative disorders, late-stage pathology may include numerous compensatory mechanisms which would make it increasingly difficult to implement a causal therapy. But substantial therapeutic benefits may still be possible, notably if basic research can reveal a (virtual) bypass in the signal flow connecting the elements of molecular intervention and motor outcome. In the present experiments with HD mice, a rather robust motor response was induced by local (striatum), cell-selective (astrocytes) and sitedirected (EAAT2 C-terminus) transgene expression. Such result is to some extent surprising because most evidence-based models describe the initiation of voluntary movements as the result of a multi-level parallel and distributed information processing in a large number of brain structures (see for instance (M orita, 2014)). That a particular cell population can effectively control a set of motor acts has mostly been implicated in "lower" vertebrates or invertebrates.

However, reports on specific links between motor performance and specific transgene expression are already accumulating (see, for instance, ( $N$ agai et al., 2019; Percie du et al., 2020)) and may help to identify new therapeutic targets. The present results have promise with regard to small molecule therapy of neurologic conditions with hypokinesia, including HD, Parkinson's disease, stroke and toxin-induced brain damage. These disorders may share similar alterations in the regulation of synaptic glutamate transport, but more information is needed for precise molecular targeting of the involved signaling cascades. The results of bidirectional testing for disease- and treatmentrelated effects and the combined use of new behavioral and cellular readouts should be a useful complement to the already existing studies on mouse models with advanced HD.

\section{Which cellular markers report failure or rescue of glutamate clearance?}

In the past glutamate uptake has mostly been quantified on the basis of tritium-labeled striatal 
tissue or synaptosomal preparations (see Introduction). In comparison with the measurement of tissue glutamate uptake, SBFI imaging of individual fluorescence-tagged astrocytes offers the advantage that the uptake activity can directly be compared in transduced vs. non-transduced astrocytes. In this way the results should be less affected by other HD-induced alterations, such as changes in the size and density of astroglia in the striatum. Our present analysis provides the still missing proof that the deficits in locomotion and synaptic glutamate clearance were in fact associated with: i) astrocytes expressing an HD phenotype, ii) the EAAT2/GLT-1 transporter and iii) signals mediated via the EAAT2-C-terminal, as opposed to changes in other cell types, transporter proteins or EAAT 2 domains.

Unfortunately, the subcellular resolution of glutamate uptake with sodium imaging is not very high, even in the case of focal glutamate uncaging (Dvorzhak et al., 2016). This limits the use of SBFI imaging for the estimation of the glutamate clearance at the sites where it matters - the tripartite synapse. Previous attempts to reveal HD-related clearance deficits with glutamate sensors (Parsons et al., 2016;Parievsky et al., 2017) al so failed, most likely due to methodical limitations (slow glutamate sensors, nonselective general tissue depolarization, low temporal and spatial resolution of synaptic responses) . Considering, however, the importance of the corticostriatal pathway for the initiation of self-induced movements (Plotkin and Surmeier, 2015) it was found worthwhile to establish a new approach for the evaluation of the glutamate transients at individual glutamatergic synapses in the dorsal striatum (Dvorzhak and Grantyn, 2019). The most telling indicators of clearance deficiency were the time constant of decay and the spread of the stimulus-induced glutamate elevation as reported by the ultrafast glutamate sensor iGlu (Helassa et al., 2018; Dvorzhak et al., 2019). It was already known that the duration of corticostriatal EPSCs increased (Dvorzhak et al., 2019) or, respectively, decreased (Lee et al., 2008; Ghosh et al., 2016) after the exposure to pharmacological blockers of glutamate transport or modulators of $N F-\kappa B$-dependent transcription. A faster decay of the glutamate elevation at corticostriatal terminals located on S506X-expressing astrocytes can therefore be regarded as a convincing argument in support of the astrocytic contribution to the glutamate clearance in HD. According to previous mathematical modeling (Zheng et al.,
2008;Scimemi and Beato, 2009;K essler, 2013) this could diminish the potentially adverse effect of extrasynaptic NM DA receptor activation.

\section{What could be the molecular basis of failing glutamate uptake in HD?}

In principle, insufficient glutamate uptake could be due to aberrant RNA splicing (Lin et al., 1998;Scott et al., 2011) or changes in the relative abundance of functional EAAT2 isoforms (Goursaud et al., 2011). A t least 3 of the latter are known to differ in their C-terminal sequence: EAAT2a, EAAT2b and EAAT2c (GLT-1a, GLT-1b and GLT 1c in rodents, see (V andenberg and Ryan, 2013) for further information). In the rat brain, EAAT2a is $\sim 15$-fold more abundant than EAAT2b (Holmseth et al., 2009). Both are found at glutamatergic synapses (A) A wabdh et al., 2016), but only EAAT2b is required for the regulated as opposed to constitutive glutamate transport (Underhill et al., 2015). The Cterminus of EAAT2b but not EAAT2a contains a sequence predicted to interact with PDZ-containing proteins, including PICK 1 (Wheatley et al., 1992;Bassan et al., 2008), PSD95 (GonzalezGonzalez et al., 2009), or DLG1(Underhill et al., 2015)). By interaction with these proteins, EAAT2 can increase its internalization and/or lateral mobility in the astrocyte membrane due to activation of CamK II (Underhill et al., 2015) or high concentration of glutamate (A) A wabdh et al., 2016). It was shown that experimental immobilization of EAAT2 prolongs synaptic currents (M urphy-Royal et al., 2015). Given that the duration of the glutamate transport cycle $(\sim 12$ ms at Schaffer collateral synapses (B ergles and Jahr, 1998)) is relatively long in comparison with the glutamate receptor binding, it was hypothesized that lateral movement of the substrate-transporter complex and a rapid membrane turnover to replenish fresh unbound transporter molecules are necessary for effective glutamate buffering at synaptic sites. To ensure the high abundance of transporter molecules around a glutamate-releasing terminal (Lehre and Danbolt, 1998) the turnover of the transporter from the cytoplasm into the membrane should also be high. However, how the degradation/replenishment of EAAT2 protein at a tripartite synapse actually works is not yet clear.

The present experiments in symptomatic Q175 HET are, to the best of our knowledge, the first attempt to characterize an HD phenotype in the EAAT 2 interactome by using immunoprecipitation of $\mathrm{mYFP}$-tagged native and C-terminal-truncated 
EAAT2 as "bait". The focus was on alterations produced by the disease, on one side, and changes due to $\mathrm{C}$-terminal truncation of EAAT2, on the other side. It became quite obvious that HD produced major changes in the abundance of fulllength EAAT2 binding partners, while expression of $M Y$ FP-EAAT2-S506X resulted in a partial return to the interaction pattern seen in $\mathrm{WT}$.

The changes in HD mice included the disappearance of normally existing, potentially necessary interactors, such as dystrophin, and the appearance of new, potentially pathological interactors, such as UBA2. Dystrophin is quite plentiful in astrocytes, especially at the endfeet, and it has been shown that dystrophin-glycoprotein complexes provide a link between laminin and the cytoskeleton (Sato et al., 2018) thereby contributing to the stabilization of aquaporin4 and Kir4.1 in particular subregions of the astrocyte plasma membrane (Enger et al., 2012). The function of dystrophin at glutamatergic corticostriatal synapses has not yet been explored, but there is evidence for a role of a dystrophin-associated protein complex in the pathogenesis of parkinsonian symptoms (Spuler et al., 2010). UBA2 and SAE 1 form a heterodimer that functions as a SUMO-activating enzyme. Sumoylation - the covalent attachment of SUM 01 to lysine residues - is a posttranslational modification process with relevance to HD (Steffan et al., 2004). Interestingly, non-sumoylated EA AT2 resides in the plasma membrane while the product of SUMO fusion with EAAT2 tends to form aggregates in the cytoplasm (Foran et al., 2011). In the present study, when comparing the UBA 2 levels precipitated by MYFP-EAAT2 or MYFP-EAAT2S506X from HET animals, it was apparent that the loss of C-terminal interaction motives largely prevented the interaction between EAAT2 and UBA2. In cultured astrocytes, a proteolytically released $C$-terminal fragment of EAAT2 modified by SUMO1 was also found in the nucleus, with inhibitory effects on EAAT2 transcription (Gibb et al., 2007; Foran et al., 2011). The relevance of this EAAT2-derived signaling mechanism was demonstrated in vivo, in a mutant SOD1 mouse model of amyotrophic lateral sclerosis (ALS), where knock-in of a modified Slcla2 isoform with a defective caspase-3 cleavage site prolonged the life span of mice afflicted by the disease (Rosenblum et al., 2017).

As for the plasma membrane localization of EAAT2, it should be noted that there is a basal constitutive internalization of the transporter, with a critical role of the lysines $497,517,526,550,558$, 570 , and 573 at the EAAT2 C-terminal (GonzalezGonzalez et al., 2008; Martinez-Villarreal et al., 2012). It is interesting that in the present SBFI imaging experiments a selective lysine-to-arginine exchange in the $\mathrm{C}$-terminal of the EAAT2 protein proved to be almost as effective as the $\mathrm{C}$-terminal truncation suggesting a critical role for $\mathrm{C}$-terminal lysine-mediated interactions such as sumoylation and ubiquitination in the regulation of glutamate uptake.

In the context of the above literature on constitutive internalization of EAAT2 and the toxic effects of sumoylated C-terminal EAAT2 cleavage products, it is tempting to propose that an astrocyte expressing $\mathrm{mHTT}$ presents with largely altered conditions for both transcriptional control and protein sorting, which perhaps explains why in Q175 HET a mere stimulation of full-length EAAT 2 failed to eliminate the deficits in the motor performance and glutamate uptake seen in HET CTRL group. Of course, much more work is needed to fully unravel the molecular determinants of EAAT2 function in the striatum and other regions of the brain.

\section{METHODS}

\section{AAV plasmid design and vector production}

Fig. 1 and Supplemental Tab. 3 present an overview of the used vectors, the sites of injections and the purpose of a chosen indicator. Plasmid PRCCMV-mYFP-EAAT2 and PRCCMVmYFP-EAAT2-S506X were gifts from Christoph Fahlke and A rnd Baumann (Forschungszentrum Jülich). pRcCM V-mY FPEAAT2 contains the expression cassette for the human SIcla2 fused to N-terminal mYFP. In PR CCM V -mYFP-EAAT2-S506X a serine at position 506 is point-mutated to generate a stop codon that causes the truncation of the last 68 amino acids of the $C$ terminus. This truncation does not interfere with the membrane insertion of the protein or glutamate transport (Leinenweber et al., 2011). In contrast, the mutation of four C-terminal lysine (Lys, K) residues to arginine $(\mathrm{Arg}, \mathrm{R})$ in the full-length EAAT2 has been shown to be critical for transporter internalization (Gonzalez-Gonzalez et al., 2008). Four consecutive cycles of site-directed mutagenesis were applied to generate EAAT2-4KR. In brief, pRcCM V-mYFP-EAAT2 was amplified by PCR using the mutagenesis primers listed in Supplemental Tab. 1, and the template was subsequently destroyed by $\mathrm{Dpnl}$ digest. For highefficiency transformation, NEB C2987H $₫$ alpha-competent $\mathrm{E}$. coli were incubated with the D pnl-digested PCR mix. Successful mutants were screened by Sanger sequencing (Eurofins Genomics, Köln). The expression cassettes for mYFP-EAAT2, mY FP-EAAT2-4KR and mYFP-EAAT2-S506X were subcloned by restriction digest with $\mathrm{BamHI}$ and $\mathrm{ECORI}$ and ligation into a preexisting AAV backbone (pAAV-gfaABC1D-ChR2(LCTC)p2a-FP635-WPRE) to produce pAAV-gfaABC1D-mYFPEAAT2-WPRE, pAAV-gfaABC1D -mYFP-EAAT2-4KRWPRE and PAAV-gfaABC1D -mYFP-EAAT2-S506X-WPRE. $M$ onomeric mRuby3 was subcloned from pKanCM V-mRuby3- 
bioRxiv preprint doi: https://doi.org/10.1101/2020.09.17.302158; this version posted March 26, 2021. The copyright holder for this preprint (which was not certified by peer review) is the author/funder. All rights reserved. No reuse allowed without permission.

10aa-H2B into the three AAV vectors with Agel and BsrGI to facilitate detection of the transduced astrocytes and the EGFP. based $\mathrm{iGlu}_{u}$ in the corticostriatal terminals of the same preparation. Control vectors were constructed by amplifying mRuby3 or mYFP by PCR adding a $3^{\prime}$ stop codon and a Sall restriction site and were then cloned into an $A A V$ backbone to generate PAAV-gfaABC1D-mYFP-WPRE or PAAVgfaA BC1D-mR uby3-WPRE. The plasmids used or modified for further use are listed in Supplemental Tab. 1. The AAV vectors were produced at the Vector Core Facility of the Charité U niversity M edicine or at U Penn V ector Core. W e acknowledge the contribution of Viviana Gradinaru and B enjamin Devermann for generating the PHP.eB adeno-associated virus (AAV) serotype (Chan et al., 2017).

\section{Antibodies}

The primary and secondary antibodies used for the quantification of $W B$ or IR are also listed in Supplemental Tab. 3. For concentrations see the Methods section "Quantitative immunofluorescence (IF)" and "Western blot analysis of striatal lysates". The EAAT2-Ab (A bcam, Cambridge, UK) was raised against a synthetic peptide within rat EAAT2 aa 550 to the Cterminus ( $C$ terminal) conjugated to keyhole limpet haemocyanin. The exact sequence is proprietary. A part from several other $C$-terminus-directed Abs, we also tried Ab77039 and Ab203130 from Abcam were peptide fragments between aa143 and 239 were used as antigen but did not achieve the quality of immunostaining seen with A b41621.

\section{Animals}

Z-Q175-KI mice were obtained from CHDI ("Cure Huntington's Disease Initiative", see stock \# 027410 of the Jackson Laboratory, Bar Harbor, USA). All applicable international, national and institutional guidelines for the care and use of the animals were followed. The study design, performance of experiments and statistical evaluation has been approved by the Berlin Office of Health Protection and Technical Safety (G0218/17), with a yearly update of the experimental guidelines by the local authorities according to the 10 essential rules of ARRIVE (see latest update from J uly 14, 2020 and (Percie du et al., 2020)). The experiments were performed in animals of either sex at an age of 51 to 76 weeks. The number of CAG repeats ranged from 182 to 201 and were determined by Laragen (Culver City, CA, USA). Care was taken that all experimental groups contained an equal number of males and females $( \pm 1)$. A part from the body weight, no systematic differences could be detected in any of the evaluated indicators. Blinding was not applied, since the animals were used at a stage of HD when the experimenters could recognize the respective genotype and select the appropriate mice among a usually very small number of available animals. The necessary sample size was kept to a minimum and was calculated beforehand to achieve hypothesis testing at a significance level of $5 \%$.

\section{Stereotaxic and intravenous injections of viral expression vectors for treatment and diagnostic purposes}

Q175 WT and HET were anaesthetized by intraperitoneal injection of a mixture containing $87.5 \mathrm{mg} / \mathrm{kg}$ ketamine and 12.5 $\mathrm{mg} / \mathrm{kg}$ xylazine (both from Sigma-Aldrich, Taufkirchen) before receiving bilateral intrastriatal injections of PHP.eB-mRuby3gfaABSID-EAAT2 (or respective isoforms/controls) as part of the treatment. The therapeutic vectors or controls were given at a concentration of $1.0^{*} 10^{12} \mathrm{gc} / \mathrm{ml}$ and an amount of $1 \mu \mathrm{l}$ per site $\left(1.0 * 10^{9} \mathrm{gc} /\right.$ striatum $)$ at the following coordinates with respect to bregma $(\mathrm{mm})$ : anterior 0.75 , lateral 2.0 , ventral 2.5 . The intravenous injections $(100 \mu \mathrm{l})$ were performed under slight isoflurane anaesthesia and adjusted to an injected amount of
$2.0^{*} 10^{11}$ to $3.5 * 10^{11} \mathrm{gc} / \mathrm{animal}$. The expression vector for the glutamate sensor AAV9-CamKII.iGluu.WPRE-hGH $\left(7.34 * 10^{13} \mathrm{gc} / \mathrm{ml}-0.3 \mu \mathrm{l}\right)$ was applied at four sites at: anterior 1.5, lateral 1.56, 1.8, 2.04, 2.28 and ventral 1.7. The expression time between injection and sacrifice was 3-5 weeks.

\section{Behavioral tests in WT and Q 175 HE T}

On the day of sacrifice, the animals were submitted to a classical open field test $(53 \mathrm{~cm} \times 33 \mathrm{~cm})$, as described in a previous study with Q175 homozygotes (Rothe et al., 2015). The mice were individually tested between 9 a.m. and 2 p.m. using an openfield box made of gray plastic with $53 \mathrm{~cm} \times 33 \mathrm{~cm}$ surface and $19 \mathrm{~cm}$ walls, illuminated by a spot of $25 \mathrm{~W}$ placed $1 \mathrm{~m}$ above the shadow-free box. M onitoring of 5 -min sessions was done by a video camera (Logitech C525 Webcam, 15 frames/s). A customary software was designed to quantify not only running activity but also the motor activity at rest. This required the calculation of the centroid of all pixels derived from the black mouse body. To this end, the background frame without mouse was subtracted from frames with mouse using an intensity threshold of mean + 3SD. A virtual mouse radius (v.m.r.) was derived from the area of suprathreshold mouse pixels approximated as a circle. The identification of the resting state is based on the time needed to cross 2 v.m.r. For more details and definitions see Supplemental text.

For the "step-over test" the animal was placed into the center of a Petri dish (diameter $18.5 \mathrm{~cm}$ and wall height $2.8 \mathrm{~cm}$ ). The movements were recorded with a video camera. Using offline analysis, a software routine determined the time between the take-off of the experimenter's hand (in a black glove) and the moment when the animal has reached the outside of the dish with all 4 feet. This parameter was called "step-over latency" and correlated with the open field total path in $5 \mathrm{~min}$.

\section{Preparation of acute brain slices}

The animals were anaesthetized with isoflurane, transcardially perfused with cooled aerated saline containing (in $\mathrm{mM}$ ): NM DG - 92, $\mathrm{KCl}-2.5, \mathrm{NaH}_{2} \mathrm{PO}_{4}-1.25, \mathrm{NaHCO}_{3}-25$, glucose - 20, $\mathrm{CaCl}_{2}-0.5, \mathrm{MgCl}_{2}-10$, sodium pyruvate -3 , and sodium ascorbate - 5 (pH 7.35, $303 \mathrm{mosmol} / \mathrm{l})$. After decapitation and removal of the brains, parasagittal (10 deg off) sections (300 $\mu \mathrm{m}$ ) containing the striatum were prepared as previously described (Dvorzhak et al., 2016). The slices were kept in artificial cerebrospinal fluid (ACSF) containing (in $\mathrm{mM}$ ): $\mathrm{NaCl}$ $125, \mathrm{KCl}-3, \mathrm{NaH}_{2} \mathrm{PO}_{4}-1.25, \mathrm{NaHCO}_{3}-25, \mathrm{CaCl}_{2}-2, \mathrm{M} \mathrm{gCl}_{2}-$

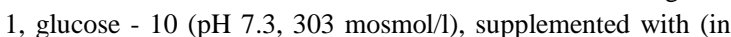
$\mathrm{mM}$ ) sodium pyruvate - 0.5 , sodium ascorbate -2.8 and glutathione -0.005 . These perfusion and recovery solutions preserved the astrocytes better than physiological ACSF, sucrose- or choline-containing solutions, the criterion being the astrocyte resting membrane potential at break-in WT $(<=-75$ $\mathrm{mV})$.

\section{Sodium imaging of astrocytes in striatal slices}

Our methods for sodium imaging in striatal astrocytes largely followed the techniques already described (Dvorzhak et al., 2016). B riefly, the slices were incubated for $20-30 \mathrm{~min}$ in oxygenated ACSF containing $222 \mu \mathrm{M}$ of the membranepermeable form of SBFI (\#S-1264, Thermo Fisher Scientific), and $2.5 \%$ dimethyl sulfoxide (DM SO) and 0.5\% Pluronic F-127 at $36^{\circ} \mathrm{C}$. For recording of the $\mathrm{L}$-aspartate-induced sodium transients, the gap junction blocker CBX (100 $\mu \mathrm{M}$, A bcam) was added to the superfusion solution, along with blockers of ionotropic glutamate receptor blockers (DNQX $10 \mu \mathrm{M}$ and MK801 $1 \mu M$, Tocris, B ristol, UK). Wide-field fluorescence imaging of SBFI-AM-stained slices was performed using a 
bioRxiv preprint doi: https://doi.org/10.1101/2020.09.17.302158; this version posted March 26, 2021. The copyright holder for this preprint (which was not certified by peer review) is the author/funder. All rights reserved. No reuse allowed without permission.

digital live acquisition imaging system (Andor Solis version 4.30.30034.0, A cal GmbH, Gröbenzell) and a sCM OS camera (AndorZyla 4.2 plus) attached to an upright Zeiss microscope (Axio Examiner A 1, Göttingen). Images were collected with a Zeiss 63x NA 1.0 water immersion plan apochromat objective. Cells were selected based on the resting levels of SBFI and mR uby3 fluorescence excited by a UVICO ultraviolet or visible light source (Rapp OptoElectronic, Hamburg), combined with suitable filter sets from Omega Optical (B rattleboro, VT, USA) attached to a FW 1000 filter wheel (Applied Scientific Instrumentation, Eugene, OR, USA). Single wavelength sodium imaging was performed by excitation of $\mathrm{SBFI}$ at $380 \mathrm{~nm}$ (sodium-sensitive wavelength). SBFI emission was collected at $>510 \mathrm{~nm}$ (dichroic mirror XF2002, emission filter XF3086, Omega Optical). Regions of interest (ROIs) with a size of 3.2 $\mu \mathrm{m} \times 3.2 \mu \mathrm{m}$ were defined on the cell body. Binning was $8 \times 8$. The spatial resolution was then $0.8 \mu \mathrm{m} /$ pixel. The exposure times was set to $150 \mathrm{~ms}$ in all experiments. Images were acquired every $3 \mathrm{sec}$. Custom-written software was used to control image acquisition and the valves operating the superfusion system. After $1 \mathrm{~min}$ of baseline recordings (20 images), L-aspartate (1 mM) was applied for 1 min followed by a $3 \mathrm{~min}$ washout period. Subsequently, the same routine was repeated in the presence of the glutamate transport blocker TFBTBOA $2 \mu \mathrm{M}$ (Tocris). For both traces the fluorescence change was calculated from the average of the baseline fluorescence intensities for each $\mathrm{ROI}$ as $\Delta \mathrm{F} / \mathrm{F}=\left(\mathrm{F}_{\mathrm{SBF}}\right.$ -

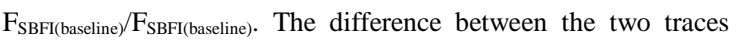
represents the L-aspartate-induced sodium transient that is mediated by all available glutamate transporters. The response to $L$-aspartate was verified by student's $t$-test comparison between response peak and baseline.

\section{G lutamate imaging at single corticostriatal synapses}

The methods established to image glutamate release from single corticostriatal presynaptic terminals and to identify synapses exhibiting an HD phenotype have already been described in some detail (Dvorzhak et al., 2019). B riefly, acute slices were submerged into a perfusion chamber with a constant flow of oxygenated ACSF at a rate of $1-2 \mathrm{ml} / \mathrm{min}$. The temperature during the recordings was maintained at $26-27{ }^{\circ} \mathrm{C}$. Single varicosities expressing the ultrafast glutamate sensor iGlu (Helassa et al., 2018) were visualized using a Zeiss wide field microscope (Axioscope 2, FS Plus) with a 63x /NA 1.0 water immersion objective and brief (180 ms) discontinuous exposure to a $473 \mathrm{~nm}$ laser beam focused to a circular area of $\sim 4.5 \mu \mathrm{m}$ in diameter. For evaluation of evoked responses, the iGluu fluorescence was acquired at a frequency of $2.5 \mathrm{kHz}$ from a rectangular ROI of $4 \mu \mathrm{m} \times 4 \mu \mathrm{m}(20 \times 20$ pixels, binning 2$)$ using a SCM OS camera (Andor Zyla4.2 plus). Laser, camera and electrical stimulation of the axon/bouton were controlled by in-house written software routines. Each pixel of the ROI was evaluated separately. The iGlu $\mathrm{u}_{\mathrm{u}}$ pixel signal was expressed as change of fluorescence intensity $\Delta F$ in $\%$ of baseline fluorescence of the given pixel. The baseline is the mean of the intensity values obtained during the $50 \mathrm{~ms}$ period prior to stimulation. For the construction of time- and space-dependent [Glu] profiles after evoked release, suprathreshold pixels were determined, the threshold being defined as $3 \mathrm{SD}$ of the $\Delta \mathrm{F} / \mathrm{F}$ baseline. The stimulus-induced changes of suprathreshold $\Delta F / F$ in time or space will be referred to as "iGlu transients" or simply "transients". The term "Peak amplitude" refers to the peak $\Delta \mathrm{F} / \mathrm{F}$ value of an averaged intensity transient derived from all suprathreshold pixels. "Tau decay" or "TauD" is the time constant of decay derived by fitting a monoexponential function to the decay from the peak of the averaged transients. The spatial extension of the iGlu $\mathrm{u}_{u}$ signal is described on the basis of a virtual diameter derived from the area of all suprathreshold pixels combined to form a virtual circle. The area of suprathreshold pixels and the resulting virtual diameter were used as indicators of "B outon size" (at rest, before stimulation ) or "Spread" (after stimulation and glutamate release). The term "Peak spread" refers to the peak value of the averaged spread transient. Dysfunctional synapses could best be detected by analysis of single-pixel $i G l u_{u}$, using the pixel with the highest $i G l u_{u}$ elevation at any given terminal. The highest iGlu elevations were always found within or next to the bouton at rest. The peak amplitude of the single pixel transient with the highest iGlu elevation are referred to as "M aximal amplitude". The respective TauD values are referred to as "TauDmax".

\section{Identification of dysfunctional synapses}

To induce the glutamate release from individual synaptic boutons under physiological conditions, a depolarizing current pulse was applied through an ACSF-filled glass pipette (tip diameter $<1 \mu \mathrm{M}$, resistance $10 \mathrm{M} \Omega$ ) placed next to an axon in close proximity with a fluorescent varicosity. Responses were elicited at minimal intensity at a repetition frequency of $0.1 \mathrm{~Hz}$. To challenge the glutamate uptake mechanisms at the site of release, individual synapses were stimulated under condition of blocked action potential generation (in the presence of tetrodotoxin, TTX, $1 \mu \mathrm{M}$ and in elevated $(5 \mathrm{mM})\left[\mathrm{Ca}^{2+}\right]_{\mathrm{ec}}$. This standardized activation mode bypassed eventually existing disease-related differences in the myelination and excitability of corticostriatal axons. The identification of an HD phenotype in corticostriatal synapses is based on the TauD value of the glutamate transient (Dvorzhak et al., 2019).

\section{Q uantitative immunofluorescence at glutamatergic synapses}

Using deep isoflurane anaesthesia, mice were transcardially perfused with $60 \mathrm{ml}$ ice-cold phosphate-buffered saline (PBS) containing $4 \%(\mathrm{w} / \mathrm{v})$ paraformaldehyde in PBS. Sagittal sections $(30 \mu \mathrm{m})$ were prepared as previously described (Rothe et al., 2015). For the quantification of the transduction rate and transduction specificity freely floating sections were triplestained with goat anti-td-Tomato (also detects RFP variants like mRuby3) 1:6000 (Sicgen-A cris, Carcavelos), mouse anti S100ß 1:2000 (Novus, A bingdon) and rabbit anti-lbal 1:1000 (Wako Chemicals $\mathrm{GmbH}$, Neuss) or double-stained with goat anti-tdTomato and mouse anti-NeuN 1:500 (Merck Millipore, Darmstadt). Counter-staining for nuclei with 426-diamidin-2phenylindol (DAPI) 1:10000 was performed in both experiments. For the quantification of synaptic EAAT2 fluorescence, the sections were triple-stained with goat antitdTomato- 1:6000 (Sicgen-Acris), guinea pig anti-vGluT1 1:1600 (Synaptic Systems, Göttingen) and rabbit anti-EAAT2 1:2000 (A bcam), followed by respective secondary antibodies, as listed in Supplemental Tab. 1. All sections from the different test groups were stained together for reliable comparison of EAAT2 IF levels.

RGB 24 bit images ( 1024 x 1024 pixels, pixel size $0.08 \mu \mathrm{m}, 3 x$ zoom, no binning) were acquired from the dorsal striatum using an inverted Leica DMI 6000 confocal microscope with an HCX PL Apo 63x oil objective (NA1.4) and stored in the tiff file format. A reas of interest (A Ols, $400 \times 400$ pixels) were cropped from the larger viewfields, selecting neuropil areas with a minimum of cell somatas or vessels. Quantification of EAAT2 IF was performed using ImagePro Plus (M ediaCybernetics, Roper, Sarasota). For comparison purposes, the same staining conditions and acquisition settings were applied to achieve reliable EAAT2 quantification in 4-5 groups with at least 4 animals per group. Within the selected $\mathrm{A} O \mathrm{Is}$, smaller rectangular ROIs ( $25 \times 25$ pixels) were centred to individual vGluT $1+$ spots 
bioRxiv preprint doi: https://doi.org/10.1101/2020.09.17.302158; this version posted March 26, 2021. The copyright holder for this preprint (which was not certified by peer review) is the author/funder. All rights reserved. No reuse allowed without permission.

to determine the level of synaptic EAAT2 IF. A threshold algorithm was used to define the boundaries of the EAAT2+ area excluding pixels with $\mathrm{F}<\mathrm{RO}$ I mean $+0.5 \mathrm{SD}$. The data is expressed as integral intensity of suprathreshold pixels. The term "Synaptic integral EAAT2 IF" refers to the mean value from 30 individually assessed ROIs within the boundaries of one transduced (i.e. mRuby3+) astrocyte.

\section{Preparation of striatal lysates}

The animals were anaesthetized with isoflurane, transcardially perfused with cooled aerated saline containing (in $\mathrm{mM}$ ): $\mathrm{N}$ methyl-D-glucamine chloride (NMDG) - $92, \mathrm{KCl}-2.5$, $\mathrm{NaH}_{2} \mathrm{PO}_{4}-1.25, \mathrm{NaHCO}_{3}-25$, glucose - 20, $\mathrm{CaCl}_{2}-0.5, \mathrm{M} \mathrm{gCl}_{2}-$ $10, \mathrm{Na}$ pyruvate -3 , and $\mathrm{Na}$ ascorbate - 5 (pH 7.35, 303 mosmol/l). If not mentioned otherwise, all chemicals and drugs mentioned here and in the following were obtained from SigmaA ldrich (Taufkirchen). B rains were quickly removed, placed into a custom-made slicing mould and immersed into ice-cold oxygenated NMDG preparation solution. $2 \mathrm{~mm}$ thick sagittal slices where cut at a distance of $1-3 \mathrm{~mm}$ from midline. The striata were dissected and snap-frozen in liquid nitrogen. The tissue was pulverized under cryogenic conditions using a cryogrinder set (CG 08-02, OPS Diagnostics, Lebanon, NJ, USA). The samples were solubilized in $200 \mu$ l lysis buffer containing (in $\mathrm{mM}$ ) NaCl 150, Tris pH 7.5 50, n-ethylmaleimide 20, dithiothreitol (DTT) 1 supplemented with glycerol 5\%, igepal ca-630 $1 \%$ and Roche complete protease inhibitor cocktail -1x (all from Sigma-Aldrich), kept on ice for $30 \mathrm{~min}$ and homogenized at $5000 \mathrm{rpm}$ using a Polytron PT1300D homogenizer. Cell debris was removed by centrifugation at $14.000 \mathrm{xg}$ for $10 \mathrm{~min}$ at $4{ }^{\circ} \mathrm{C}$

The cleared lysates were either directly submitted to WB analysis or used for the immunoprecipitation of YFP-tagged EAAT2 variants with magnetic beads for subsequent WB analysis or mass spectrometry.

\section{Western blot analysis of striatal lysates}

Samples were prepared according to the NuPAGE Technical Guide of Invitrogen. B riefly, after denaturation in NuPA GE LDS sample buffer with DTT $50 \mathrm{mM}$ for $10 \mathrm{~min}$ at $70^{\circ} \mathrm{C}$ the samples and markers were run on a Novex bis-tris gradient gel (4-12\%, Thermo Fischer Scientific) using NuPA GE M OPS SDS running buffer and subsequently blotted on a Novex $0.45 \mu \mathrm{m}$ nitrocellulose membrane (LC2001, Thermo Fischer Scientific). The membranes were washed and blocked in ReadyTector solution A (CANDOR Bioscience $\mathrm{GmbH}$, Wangen), and the primary antibodies rabbit anti-EAAT2 1:2000 (Abcam, Cambridge, UK) and mouse anti-GAPDH 1:1000 (SigmaAldrich) were directly applied for $1 \mathrm{~h}$ at room temperature in solution $B$ that also contained horseradish peroxidase (HRP) coupled to the secondary antibodies against rabbit or mouse, respectively. To detect $\mathrm{mYFP}$, membranes were blocked for 30 min in 1x Roti-block solution (Carl Roth $\mathrm{GmbH}$, Karlsruhe) before applying rabbit anti-GFP 1:1000 (ChromoTek GmbH, $\mathrm{M}$ artinsried) over night at $4{ }^{\circ} \mathrm{C}$ in the same solution. Then the HRP-coupled secondary antibodies (Dianova GmbH, Hamburg) were applied at 1:2500 for $3 \mathrm{~h}$ at $4{ }^{\circ} \mathrm{C}$. Proteins were detected using respective kits from Biozym Scientific $\mathrm{GmbH}$ (Hessisch Oldendorf . Proteins were detected by chemiluminescence and submitted to image analysis with Imagej .

\section{Immunoprecipitation of YFP-tagged EAAT2 for western blotting or mass spectrometry}

Striatal lysates were diluted with $300 \mu \mathrm{l}$ wash buffer (in mM) $\mathrm{NaCl} 150$, Tris pH 7.550 , DTT 1, glycerol $5 \%$ and incubated for $2 \mathrm{~h}$ with $30 \mu \mathrm{l}$ of pre-washed GFP-Trap magnetic agarose beads (gtma-20, ChromoTek, Planegg-M artinsried) using gentle rotation at $4{ }^{\circ} \mathrm{C}$. The beads-protein complexes were isolated using a DynaM ag-2 magnet (Thermo Fisher Scientific), applying 3 wash-resuspension cycles before short-term storage of the immunoprecipitate at $-18{ }^{\circ} \mathrm{C}$. The first wash solution contained igepal ca- $6300.05 \%$. For WB analysis the beads were for $10 \mathrm{~min}$ incubated in the NUPAGE LDS sample buffer and treated as described in the previous chapter. For liquid chromatography tandem mass spectrometry (LC-MS), the beads were first incubated in digestion buffer (sodium deoxycholate - $1 \%$, dithiothreitol $-10 \mathrm{mM}$, ammonium bicarbonate $-50 \mathrm{mM}, 45$ min, r. t.). The proteins were then submitted to alkylation with $55 \mathrm{mM}$ chloroacetamide (30 min, r. t. complete darkness) and over-night digestion with $500 \mathrm{ng}$ endopeptidase LysC (Wako, $\mathrm{N}$ euss) and $500 \mathrm{ng}$ sequence grade trypsin (Promega, M annheim, GER) at $37^{\circ} \mathrm{C}$. The samples were then acidified with formic acid (final concentration $1 \%$ ).

\section{EAAT 2 interaction proteomics}

The peptides were extracted and desalted using the StageTips protocol. Separation was carried out using in-housemanufactured $20 \mathrm{~cm}$ fritless silica microcolumns with an inner diameter of $75 \mu \mathrm{m}$, packed with ReproSil-Pur C18-A Q $1.9 \mu \mathrm{m}$ resin (Dr. M aisch G mbH, A mmerbuch), a 98 min gradient with a $250 \mathrm{nl} / \mathrm{min}$ flow rate of increasing Buffer $B$ concentration (from $2 \%$ to $60 \%$, Buffer B: $90 \%$ acetonitrile) on an High Performance Liquid Chromatography (HPLC) system from Thermo Fischer Scientific. The eluting peptides were directly ionized by electrospray ionization and transferred into a Thermo Orbitrap Fusion mass spectrometer. The instrument was operated in the data-dependent mode with performing full scans in Orbitrap (60K resolution; $4 \times 10^{5}$ ion count target; maximum injection time $50 \mathrm{~ms}$ ), followed by top $20 \mathrm{M} \mathrm{S2}$ scans using higher-energy collision dissociation (NCE of 32; 15K resolution, $5 \times 10^{4}$ ion count target; $0.7 \mathrm{~m} / \mathrm{z}$ isolation window; maximum injection time: $250 \mathrm{~ms}$ ). Only precursor with charge states between 2-7 were fragmented. Dynamic exclusion was set to $30 \mathrm{sec}$. Raw data were analyzed using the MaxQuant software (v1.6.0.1). The internal Andromeda search engine was used to search MS2 spectra against a decoy UniProt database for mouse (M OUSE.2018-05), as well as the sequences of the mY FP fusion constructs, containing forward and reverse sequences. The search included variable modifications of oxidation (M) and $\mathrm{N}$ terminal acetylation, deamidation ( $N$ and $Q$ ) and fixed modification of carbamidomethyl cysteine. Minimal peptide length was set to 7 amino acids and a maximum of two missed cleavages was allowed. The false discovery rate (FDR) was set to 0.01 for peptide and protein identifications. The integrated label-free quantification and IBAQ calculation algorithm were activated. Unique and razor peptides were considered for quantification. Retention times were recalibrated based on the built-in nonlinear time-rescaling algorithm and MS/MS identifications were transferred between LC-M S/M S runs with the "Match between runs" option, in which the maximal retention time window was set to $0.7 \mathrm{~min}$. The resulting text files were used for further analyses using the Perseus software package (omicX, v. 1.6.2.1). LFQ intensity values were used for quantification. Reverse hits, contaminants and proteins only identified by site were filtered out. Technical and biological replicates for each condition were defined as groups and intensity values were filtered for "minimum value of 3 " per group. After log2 transformation missing values were imputed with random noise simulating the detection limit of the mass spectrometer. Imputed values are taken from a log normal distribution with $0.25 \times$ the standard deviation of the measured, logarithmized values, down-shifted by 1.8 standard deviations. The data was obtained from 4 test groups, each comprising at least 4 animals per group. The signals obtained from any given 
bioRxiv preprint doi: https://doi.org/10.1101/2020.09.17.302158; this version posted March 26, 2021. The copyright holder for this preprint (which was not certified by peer review) is the author/funder. All rights reserved. No reuse allowed without permission.

animal (pooling the tissue from both striata) were normalized to the mYFP signal median intensity calculated from all samples. Only proteins with $>3$ peptide detection and abundance of log2 intensities $>=23$ in all 4 samples were included. Differential protein abundance was calculated using two-sample Student's t test, applying a permutation-based FDR cut-off at $1 \%$. A bundance differences between the samples with a $p$-value of $<=0.05$ in a two-tailed t-test were considered significant.

\section{Data evaluation and statistical analysis}

The comparison of the means could be influenced by interanimal variance. Therefore, in the case that individual sections, cells or synapses were obtained from different animals, multilevel ("nested data") analysis was performed with Prism 8
(GraphPad, San Diego, USA). P values of $<0.05$ were considered statistically significant. Differences between the groups were tested with ANOVA or respective nonparametric methods (K ruskal-Wallis-test), followed by multiple comparison (Dunnet's or Dunn's tests). Significance levels were marked by asterisks, where * corresponds to $\mathrm{P}<0.05, * *-\mathrm{P}<0.01$ and $* * *$. $P<0.001$. The HET-CTRL data served as reference for comparison with WT-CTRL or HET-TEST. Effect strength was described according to Cohen's D or Hedges' G. D or G values larger 0.8 suggest that the respective effect was strong. 


\section{R eference $L$ ist}

Al A wabdh S, Gupta-A garwal S, Sheehan DF, M uir J, N orkett R, Twelvetrees A E, Griffin LD, Kittler JT (2016) Neuronal activity mediated regulation of glutamate transporter GLT-1 surface diffusion in rat astrocytes in dissociated and slice cultures. Glia 64: 1252-1264.

A rkhipova V, Trinco G, Ettema TW, Jensen S, Slotboom DJ, Guskov A (2019) Binding and transport of Daspartate by the glutamate transporter homolog GIt(TK). Elife 8: e45286.

Bassan M, Liu H, Madsen KL, Armsen W, Zhou J, Desilva T, Chen W, Paradise A, Brasch M A, Staudinger J, Gether U, I rwin N, Rosenberg PA (2008) Interaction between the glutamate transporter GLT Ib and the synaptic PDZ domain protein PICK 1. Eur J N eurosci 27: 66-82.

B eart PM, O'Shea RD (2007) Transporters for L-glutamate: an update on their molecular pharmacology and pathological involvement. Br J Pharmacol 150: 5-17.

B ehrens PF, Franz P, Woodman B, Lindenberg KS, Landwehrmeyer GB (2002) Impaired glutamate transport and glutamate-glutamine cycling: downstream effects of the Huntington mutation. Brain 125: 19081922.

B erardelli A, N oth J, Thompson PD, B ollen EL, Curra A, Deuschl G, van Dijk J G, Topper R, Schwarz M, Roos RA (1999) Pathophysiology of chorea and bradykinesia in Huntington's disease. M ov Disord 14: 398403.

B ergles DE, Jahr CE (1998) Glial contribution to glutamate uptake at Schaffer collateral- commissural synapses in the hippocampus. J Neurosci 18: 7709-7716.

B radford J, Shin JY, Roberts M, W ang CE, Li XJ, Li S (2009) Expression of mutant huntingtin in mouse brain astrocytes causes age-dependent neurological symptoms. Proc Natl A cad Sci U S A 106: 22480-22485.

B urgold J, Schulz-Trieglaff EK, V oelkl K, Gutierrez-A ngel S, Bader J M, Hosp F, M ann M, A rzberger T, K lein R, Liebscher S, Dudanova I (2019) Cortical circuit alterations precede motor impairments in Huntington's disease mice. Sci Rep 9: 6634-43024.

Carrillo-Reid L, Day M , X ie Z, M elendez AE, Kondapalli J, Plotkin J L, W okosin DL, Chen Y, K ress GJ , K aplitt M, Ilijic E, Guzman JN, Chan CS, Surmeier DJ (2019) Mutant huntingtin enhances activation of dendritic Kv4 K (+) channels in striatal spiny projection neurons. Elife 8. pii: 40818.

Chan KY, Jang M J, Y oo B B, Greenbaum A, Ravi N, W u WL, SÃ inchez-Guardado L, L ois C, M azmanian SK, Deverman BE, Gradinaru V (2017) Engineered AAV s for efficient noninvasive gene delivery to the central and peripheral nervous systems. $N$ at N eurosci 20: 1172-1179.

Danbolt NC (2001) Glutamate uptake. Prog N eurobiol 65: 1-105.

Deng Y P, Wong T, B ricker-A nthony C, Deng B, Reiner A (2013) Loss of corticostriatal and thalamostriatal synaptic terminals precedes striatal projection neuron pathology in heterozygous Q140 Huntington's disease mice. Neurobiol Dis 60: 89-107.

Dvorzhak A, Grantyn R (2019) Single synapse indicators of glutamate release and uptake in acute brain slices from normal and Huntington mice. J $V$ is Exp.

Dvorzhak A , Helassa N, Torok K, Schmitz D, Grantyn R (2019) Single synapse indicators of impaired glutamate clearance derived from fast iGluu imaging of cortical afferents in the striatum of normal and Huntington (Q175) mice. J Neurosci 39: 3970-3982.

Dvorzhak A, Vagner T, Grantyn R (2016) Functional indicators of glutamate transport in single striatal astrocytes and the influence of $\mathrm{K} \mathrm{ir} 4.1$ in normal and Huntington mice. J Neurosci 16: 4959-4975.

Enger R, Gundersen GA, Haj-Y asein N N, Eilert-OIsen M, Thoren AE, Vindedal GF, Petersen PH, Skare 0 , Nedergaard M, Ottersen OP, Nagelhus EA (2012) Molecular scaffolds underpinning macroglial polarization: an analysis of retinal M uller cells and brain astrocytes in mouse. Glia 60: 2018-2026.

Estrada-Sanchez A M , Blake CL, B arton SJ , Howe A G, R ebec GV (2019) Lack of mutant huntingtin in cortical efferents improves behavioral inflexibility and corticostriatal dynamics in Huntington's disease mice. J Neurophysiol 10.

Estrada-Sanchez A M, M ontiel T, Segovia J, M assieu L (2009) Glutamate toxicity in the striatum of the R6/2 Huntington's disease transgenic mice is age-dependent and correlates with decreased levels of glutamate transporters. Neurobiol Dis 34: 78-86.

Faideau M, Kim J, Cormier K, Gilmore R, Welch M, Auregan G, Dufour N, Guillermier M, B rouillet E, Hantraye P, D eglon N, Ferrante RJ, B onvento $G$ (2010) In vivo expression of polyglutamine-expanded huntingtin by mouse striatal astrocytes impairs glutamate transport: a correlation with Huntington's disease subjects. Hum M ol Genet 19: 3053-3067.

Foran E, Bogush A, Goffredo M, Roncaglia P, Gustincich S, Pasinelli P, Trotti D (2011) Motor neuron impairment mediated by a sumoylated fragment of the glial glutamate transporter EAAT2. Glia 59: 1719-1731.

Ghosh M, Lane M, Krizman E, Sattler R, Rothstein JD, Robinson M B (2016) The transcription factor Pax6 contributes to the induction of GLT-1 expression in astrocytes through an interaction with a distal enhancer element. J N eurochem 136: 262-275. 
bioRxiv preprint doi: https://doi.org/10.1101/2020.09.17.302158; this version posted March 26,2021 . The copyright holder for this preprint (which was not certified by peer review) is the author/funder. All rights reserved. No reuse allowed without permission.

Gibb SL, B oston-Howes W, Lavina ZS, Gustincich S, Brown RH, Jr., Pasinelli P, Trotti D (2007) A caspase-3cleaved fragment of the glial glutamate transporter EAAT2 is sumoylated and targeted to promyelocytic leukemia nuclear bodies in mutant SOD 1-linked amyotrophic lateral sclerosis. J B iol Chem 282: 3248032490.

Gonzalez-Gonzalez IM , Garcia-T ardon N, Gimenez C, Zafra F (2008) PK C-dependent endocytosis of the GLT I glutamate transporter depends on ubiquitylation of lysines located in a C-terminal cluster. Glia 56: 963974.

Gonzalez-Gonzalez IM, Garcia-Tardon N, Gimenez C, Zafra F (2009) Splice variants of the glutamate transporter GLT 1 form hetero-oligomers that interact with PSD-95 and N M DA receptors. J N eurochem 110: 264-274.

Goursaud S, Focant M C, B erger JV, Nizet Y, M aloteaux J M, Hermans E (2011) The VPAC2 agonist peptide histidine isoleucine (PHI) up-regulates glutamate transport in the corpus callosum of a rat model of amyotrophic lateral sclerosis (hSOD 1G 93A) by inhibiting caspase-3 mediated inactivation of GLT-1a. FASE B J 25: 3674-3686.

Hart EP, M arinus J, B urgunder J M, B entivoglio AR, Craufurd D, Reilmann R, Saft C, Roos RA (2013) B etter global and cognitive functioning in choreatic versus hypokinetic-rigid Huntington's disease. Mov Disord 28: 1142-1145.

Helassa N, Durst CD, Coates C, Kerruth S, A rif U, Schulze C, W iegert JS, Geeves M, Oertner TG, Torok K (2018) UItrafast glutamate sensors resolve high-frequency release at Schaffer collateral synapses. Proc Natl Acad Sci U S A 115: 5594-5599.

Holmseth S, Scott HA, Real K, Lehre K P, Leergaard TB, Bjaalie JG, D anbolt NC (2009) The concentrations and distributions of three C-terminal variants of the GLT1 (EAAT2; slc1a2) glutamate transporter protein in rat brain tissue suggest differential regulation. Neuroscience 162: 1055-1071.

Horton M C, Nopoulos P, Nance M, Landwehrmyer GB, Barker RA, Squitieri F, B urgunder JM, Quarrell 0 (2019) Assessment of the performance of a modified motor scale as applied to juvenile onset Huntington's disease. J Huntingtons Dis 8: 181-193.

Huang K, K ang M H, A skew C, Kang R, Sanders SS, Wan J, Davis NG, Hayden M R (2010) Palmitoylation and function of glial glutamate transporter-1 is reduced in the Y A C128 mouse model of Huntington disease. Neurobiol Dis 40: 207-215.

Kaemmerer WF, Grondin RC (2019) The effects of huntingtin-lowering: what do we know so far? Degener Neurol Neuromuscul Dis 9: 3-17.

K alandadze A, Wu Y, Robinson M B (2002) Protein kinase $C$ activation decreases cell surface expression of the GLT-1 subtype of glutamate transporter. Requirement of a carboxyl-terminal domain and partial dependence on serine 486.J Biol Chem 277: 45741-45750.

K essler JP (2013) Control of cleft glutamate concentration and glutamate spill-out by perisynaptic glia: uptake and diffusion barriers. PL OS One 8: e70791.

Kirschner MA, Copeland NG, Gilbert DJ, Jenkins NA, A mara SG (1994) Mouse excitatory amino acid transporter EAAT2: isolation, characterization, and proximity to neuroexcitability loci on mouse chromosome 2. Genomics 24: 218-224.

Lee SG, Su ZZ, Emdad L, Gupta P, Sarkar D, Borjabad A, Volsky DJ, Fisher PB (2008) M echanism of ceftriaxone induction of excitatory amino acid transporter-2 expression and glutamate uptake in primary human astrocytes. J Biol Chem 283: 13116-13123.

Lehre KP, Danbolt NC (1998) The number of glutamate transporter subtype molecules at glutamatergic synapses: Chemical and stereological quantification in young adult rat brain. J Neurosci 18: 8751-8757.

Leinenweber A, M achtens JP, B egemann B, Fahlke C (2011) Regulation of glial glutamate transporters by Cterminal domains. J Biol Chem 286: 1927-1937.

Lievens JC, Woodman B, M ahal A, Spasic-Boscovic O, Samuel D, Kerkerian-Le Goff L, Bates GP (2001) Impaired glutamate uptake in the R6 Huntington's disease transgenic mice. Neurobiol Dis 8: 807-821.

Lin CL, Bristol LA, Jin L, Dykes-Hoberg M, Crawford T, Clawson L, Rothstein JD (1998) A berrant RNA processing in a neurodegenerative disease: the cause for absent EAAT2, a glutamate transporter, in amyotrophic lateral sclerosis. Neuron 20: 589-602.

M artinez-Villarreal J, Garcia TN, Ibanez I, Gimenez C, Zafra F (2012) Cell surface turnover of the glutamate transporter GLT-1 is mediated by ubiquitination/deubiquitination. Glia 60: 1356-1365.

M enalled LB, K udwa A E, M iller S, Fitzpatrick J, W atson-J ohnson J, K eating N, Ruiz M, M ushlin R, A losio W, M cConnell K, Connor D, M urphy C, Oakeshott S, K wan M, B eltran J, Ghavami A, B runner D, Park LC, Ramboz S, Howland D (2012) Comprehensive behavioral and molecular characterization of a new knock-in mouse model of Huntington's disease: ZQ175. PL oS One 7: e49838.

M erienne N, V achey G, de L ongprez L, M eunier C, Zimmer V, Perriard G, Canales M, M athias A, Herrgott L, B eltraminelli $T, M$ aulet $A$, Dequesne $T$, Pythoud $C$, Rey $M$, Pellerin $L, B$ rouillet $E$, Perrier $A L$, du $P R$, Deglon N (2017) The Self-Inactivating K amiCas9 System for the Editing of CN S Disease Genes. Cell Rep 20: 2980-2991. 
bioRxiv preprint doi: https://doi.org/10.1101/2020.09.17.302158; this version posted March 26,2021 . The copyright holder for this preprint (which was not certified by peer review) is the author/funder. All rights reserved. No reuse allowed without permission.

Meunier C, M erienne N, J olle C, Deglon N, Pellerin L (2016) A strocytes are key but indirect contributors to the development of the symptomatology and pathophysiology of Huntington's disease. Glia 64: 1841-1856.

M iller BR, Dorner JL, B unner KD, Gaither TW, K lein EL, Barton SJ, Rebec GV (2012) U p-regulation of GLT1 reverses the deficit in cortically evoked striatal ascorbate efflux in the $R 6 / 2$ mouse model of Huntington's disease. J Neurochem 121: 629-638.

M orita K (2014) Differential cortical activation of the striatal direct and indirect pathway cells: reconciling the anatomical and optogenetic results by using a computational method. J N europhysiol 112: 120-146.

M orland C, Nordengen K, Larsson M, Prolo LM, Farzampour Z, Reimer RJ, Gundersen V (2013) Vesicular uptake and exocytosis of L-aspartate is independent of sialin. FA SEB J 27: 1264-1274.

M rzljak L, M unoz-Sanjuan I (2015) Therapeutic Strategies for Huntington's Disease. Curr Top B ehav Neurosci 22: 161-201.

Mullard A (2019) Pioneering antisense drug heads into pivotal trials for Huntington disease. Nat Rev Drug Discov 18: 161-163.

M urphy-Royal C, Dupuis JP, Varela J A, Panatier A, Pinson B, Baufreton J, Groc L, Oliet SH (2015) Surface diffusion of astrocytic glutamate transporters shapes synaptic transmission. $N$ at N eurosci 18: 219-226.

Nagai J, Rajbhandari AK, Gangwani M R, Hachisuka A, Coppola G, M asmanidis SC, Fanselow M S, K hakh BS (2019) Hyperactivity with Disrupted A ttention by Activation of an A strocyte Synaptogenic Cue. Cell 177: $1280-1292$.

Parievsky A, M oore C, Kamdjou T, Cepeda C, M eshul CK, Levine M S (2017) Differential electrophysiological and morphological alterations of thalamostriatal and corticostriatal projections in the R6/2 mouse model of Huntington's disease. Neurobiol Dis 108: 29-44.

Parsons MP, Vanni MP, Woodard CL, Kang R, M urphy TH, Raymond LA (2016) Real-time imaging of glutamate clearance reveals normal striatal uptake in Huntington disease mouse models. $\mathrm{N}$ at Commun 7: 11251.

Percie du SN, A hluwalia A, A lam S, A vey M T, Baker M, B rowne WJ, Clark A, Cuthill IC, Dirnagl U, Emerson M, Garner P, Holgate ST, Howells DW, Hurst V, Karp NA, Lazic SE, Lidster K, M acCallum CJ, M acleod M, Pearl EJ, Petersen OH, Rawle F, Reynolds P, Rooney K, Sena ES, Silberberg SD, Steckler T, WÃ $\tilde{A}^{1 / 4}$ rbel H (2020) Reporting animal research: Explanation and elaboration for the ARRIVE guidelines 2.0. PL oS B iol 18: e3000411.

Petr GT, Schultheis LA, Hussey K C, Sun Y, Dubinsky J M, A oki C, Rosenberg PA (2013) Decreased expression of GLT-1 in the R6/2 model of Huntington's disease does not worsen disease progression. Eur J Neurosci 38: 2477-2490.

Plotkin J L, Day M , Peterson JD, X ie Z, K ress GJ, Rafal ovich I, K ondapalli J, Gertler TS, Flajolet M, Greengard P, Stavarache M, Kaplitt M G, Rosinski J, Chan CS, Surmeier DJ (2014) Impaired TrkB receptor signaling underlies corticostriatal dysfunction in Huntington's disease. Neuron 83: 178-188.

Plotkin JL, Surmeier DJ (2015) Corticostriatal synaptic adaptations in Huntington's disease. Curr Opin Neurobiol 33C: 53-62.

Reiner A, Deng Y P (2018) Disrupted striatal neuron inputs and outputs in Huntington's disease. CNS Neurosci Ther 24: 250-280.

Rosenblatt A, A bbott MH, Gourley LM, Troncoso J C, M argolis RL, Brandt J, Ross CA (2003) Predictors of neuropathological severity in 100 patients with H untington's disease. A nn N eurol 54: 488-493.

Rosenblum LT, Shamamandri-M arkandaiah S, Ghosh B, Foran E, Lepore AC, Pasinelli P, Trotti D (2017) $M$ utation of the caspase-3 cleavage site in the astroglial glutamate transporter EAAT2 delays disease progression and extends lifespan in the SOD 1-G93A mouse model of A LS. Exp N eurol 292: 145-153.

Rothe T, D eliano M, W ojtowicz A M, Dvorzhak A, Harnack D, Paul S, V agner T, M elnick I, Stark H, Grantyn R (2015) Pathological gamma oscillations, impaired dopamine release, synapse loss and reduced dynamic range of unitary glutamatergic synaptic transmission in the striatum of hypokinetic Q175 Huntington mice. Neurosci 311: 519-538.

Sato J, Horibe S, Kawauchi S, Sasaki N, Hirata KI, Rikitake Y (2018) Involvement of aquaporin-4 in lamininenhanced process formation of mouse astrocytes in 2D culture: Roles of dystroglycan and alphasyntrophin in aquaporin-4 expression. J Neurochem 147: 495-513.

Scimemi A, B eato M (2009) Determining the neurotransmitter concentration profile at active synapses. Mol Neurobiol 40: 289-306.

Scott HA, Gebhardt FM, Mitrovic AD, Vandenberg RJ, Dodd PR (2011) Glutamate transporter variants reduce glutamate uptake in A Izheimer's disease. Neurobiol A ging 32: 553-11.

Sharma K, Schmitt S, B ergner CG, Tyanova S, Kannaiyan N, M anrique-H oyos N, K ongi K, Cantuti L, Hanisch UK, Philips MA, Rossner M J, M ann M, Simons M (2015) Cell type- and brain region-resolved mouse brain proteome. Nat N eurosci 18: 1819-1831.

Shin JY, Fang ZH, Y u ZX, Wang CE, Li SH, Li XJ (2005) Expression of mutant huntingtin in glial cells contributes to neuronal excitotoxicity. J Cell Biol 171: 1001-1012. 
bioRxiv preprint doi: https://doi.org/10.1101/2020.09.17.302158; this version posted March 26,2021 . The copyright holder for this preprint (which was not certified by peer review) is the author/funder. All rights reserved. No reuse allowed without permission.

Spuler S, K rug H, K lein C, M edialdea IC, Jakob W, Ebersbach G, Gruber D, H offmann KT, Trottenberg T, Kupsch A (2010) Myopathy causing camptocormia in idiopathic Parkinson's disease: a multidisciplinary approach. M ov Disord 25: 552-559.

Steffan JS, A grawal N, Pallos J, Rockabrand E, Trotman LC, Slepko N, Illes K, Lukacsovich T, Zhu YZ, Cattaneo E, Pandolfi PP, Thompson LM, Marsh JL (2004) SUMO modification of huntingtin and Huntington's disease pathology. Science 304: 100-104.

Tabrizi SJ , Ghosh R, Leavitt BR (2019) Huntingtin lowering strategies for disease modification in Huntington's disease. Neuron 101: 801-819.

Tabrizi SJ, Scahill RI, Owen G, Durr A, Leavitt BR, Roos RA, Borowsky B, Landwehrmeyer B, Frost C, Johnson H, Craufurd D, Reilmann R, Stout JC, Langbehn DR (2013) Predictors of phenotypic progression and disease onset in premanifest and early-stage Huntington's disease in the TRACK -HD study: analysis of 36-month observational data. Lancet N eurol 12: 637-649.

Tong X, A O Y, Faas GC, N waobi SE, X U J, Haustein M D, A nderson M A, M ody I, Olsen M L, Sofroniew M V, Khakh BS (2014) Astrocyte Kir4.1 ion channel deficits contribute to neuronal dysfunction in Huntington's disease model mice. Nat Neurosci 17: 694-703.

Underhill SM, Wheeler DS, A mara SG (2015) Differential regulation of two isoforms of the glial glutamate transporter EAAT2 by DLG1 and CaM K II. J Neurosci 35: 5260-5270.

V andenberg RJ, Ryan RM (2013) M echanisms of glutamate transport. Physiol Rev 93: 1621-1657.

Veldman M B, Y ang XW (2018) Molecular insights into cortico-striatal miscommunications in Huntington's disease. Curr O pin Neurobiol 48: 79-89.

V onsattel JP, DiFiglia M (1998) Huntington disease. J Neuropathol Exp Neurol 57: 369-384.

Wheatley SC, Suburo A M, Horn DA, V ucicevic V, Terenghi G, Polak J M, Latchman DS (1992) Redistribution of secretory granule components precedes that of synaptic vesicle proteins during differentiation of a neuronal cell line in serum-free medium. N eurosci 51: 575-582.

Zeitler B, Froelich S, M arlen K, Shivak DA, Y u Q, Li D, Pearl J R, M iller J C, Zhang L, Paschon DE, Hinkley SJ, A nkoudinova I, Lam S, G uschin D, K opan L, Cherone JM, N guyen HB, Qiao G, A taei $Y, M$ endel M C, A mora R, Surosky R, Laganiere J, V u BJ, N arayanan A, Sedaghat Y, Tillack K, Thiede C, Gartner A, K wak S, Bard J, M rzljak L, Park L, Heikkinen T, Lehtimaki KK, Svedberg M M, Haggkvist J, Tari L, Toth M, V arrone A, Halldin C, Kudwa AE, Ramboz S, Day M, Kondapalli J, Surmeier DJ, U rnov FD, Gregory PD, Rebar EJ, M unoz-Sanjuan I, Zhang HS (2019) Allele-selective transcriptional repression of mutant HTT for the treatment of H untington's disease. Nat M ed 25: 1131-1142.

Zheng K, Scimemi A, Rusakov DA (2008) Receptor actions of synaptically released glutamate: the role of transporters on the scale from nanometers to microns. Biophys ] 95: 4584-4596. 